EUROPEAN LABORATORY FOR PARTICLE PHYSICS

CERN-PPE / 97-32

4 March 1997

\title{
NEW OBSERVATIONS WITH THE GAS ELECTRON MULTIPLIER (GEM)
}

R. Bouclier, W. Dominik, M. Hoch, J.-C. Labbé, G. Million, L. Ropelewski and F. Sauli

(CERN, Geneva, Switzerland)

\author{
A. Sharma \\ (GRPHE, Université de Haute Alsace, Mulhouse, France) \\ G. Manzin \\ (Laboratori Nazionali INFN, Legnaro, Italy)
}

\begin{abstract}
We describe recent measurements realized with the Gas Electron Multiplier (GEM) mesh added as pre-amplification element to a multiwire and a microstrip chamber. Large, stable combined gains are obtained, with good uniformity and energy resolution, in a wide range of filling gases including non-flammable mixtures; coupled to a micro-strip plate, the pre-amplification element allows to maintain the high rate capability and resolution at considerably lower operating voltages, completely eliminating discharge problems. Charge gains are large enough to allow detection of signals in the ionization mode on the last element, permitting the use of a simple printed circuit as read-out electrode; two-dimensional read-out can then be easily implemented. The absence of charge multiplication in the last stage avoids charge build-up on the substrate and prevents ageing phenomena. A new generation of simple, reliable and cheap fast position sensitive detectors seems at hand.
\end{abstract}

Submitted to Nuclear Instruments and Methods in Physics Research 


\section{INTRODUCTION}

The recently introduced Gas Electron Multiplier (GEM [1]) consists of a thin polymer foil, metal-clad on both sides and perforated by a regular pattern of holes, very closely spaced; inserted in a gas volume and with the application of suitable potentials, it allows an effective pre-amplification of electrons released by ionization and drifting in the high field through the open channels. In an early work, a small size GEM grid coupled to a standard Multiwire Proportional Chamber (MWPC) was used to demonstrate the operating principle, but due to the insufficient thickness of the insulator $(25 \mu \mathrm{m})$ only moderate gains could be obtained [2]. More effective GEM grids, both in size $\left(\sim 100 \mathrm{~cm}^{2}\right)$ and thickness $(50 \mu \mathrm{m})$ have since been manufactured and used in several set-ups, including as main amplification element a standard MWPC or a Micro-Strip Gas Chamber (MSGC). With the improved design, GEM meshes provide pre-amplification factors well above 100, thus making detection of a small amount of charge in the second amplifying element amazingly easy.

This note describes the results of measurements realized with various structures in a range of conditions and operating gases, and suggests possible uses and applications of the new devices.

\section{THE GAS ELECTRON MULTIPLIER}

The basic structure of a GEM mesh consists of a thin insulating foil, metal-clad on both sides and chemically etched after photo-lithographic patterning with a regular matrix of holes, typically $100 \mu \mathrm{m}$ in diameter at the surface (Fig. 1a). Due to the manufacturing process used, the open channels have a double conical profile, and are narrower in the center of the insulator. The width of the openings (Fig. 1b) can be adjusted by controlling the parameters of fabrication.

Upon application of a difference of potential between the electrodes, a dipole field develops in the channels as shown in Fig. 2 (computed with a commercial program ${ }^{1}$ ). With $\sim 500 \mathrm{~V}$ applied (typical for operation, see below) and with symmetric, moderate external drift fields $\left(4 \mathrm{kVcm}^{-1}\right.$ in the example), the field in the central region of the channel exceeds $40 \mathrm{kVcm}^{-1}$ (Fig. 3), sufficient to induce charge multiplication for electrons drifting through. The high field, and therefore the gain, are affected very little by the value of the external potentials. From known values of the Townsend coefficient in quencher-rich gases [3] one can estimate integrated charge gains of several hundred for a channel length between 50 and $100 \mu \mathrm{m}$. Restricted by the immediate availability of materials (good quality, double side metal clad polymer), all tests here described have been realized with GEM meshes having $18 \mu \mathrm{m}$ thick copper electrodes plated on $50 \mu \mathrm{m}$ kapton; a thicker insulator (75 to $100 \mu \mathrm{m}$ ) would probably provide larger gains and be mechanically stiffer, thus easing the assembly of detectors. For the present thickness, a hole diameter at the metal sides between 80 and $120 \mu \mathrm{m}$ has been found safe to etch, with

\footnotetext{
${ }^{1}$ MAXWELL Electric Field Simulator, Ansoft Corporation. Field lines have been drawn manually.
} 
openings at the center of the polymer foil between 40 and $80 \mu \mathrm{m}$; the width of the holes is controlled by the duration and the conditions of etching. As will be discussed later, meshes with narrower holes exhibit a moderate dynamic gain shift after power on, presumably due to charge deposition on the walls. Early models had a pitch (distance between centers of the channels) of $200 \mu \mathrm{m}$ (GEM2), but in an improved design (GEM3) we have reduced this distance to $140 \mu \mathrm{m}$ in order to obtain a finer grid and reduce cross-modulations with the second amplifying structure; we have not observed major differences of behavior between the two geometries, except for a small shift in the operating voltages. As seen in the pictures, square holes with rounded angles have been preferred in order to maximize optical transparency, at the same time avoiding sharp edges. The active area $\left(100 \times 93 \mathrm{~mm}^{2}\right.$ in the larger GEM) is surrounded by a few $\mathrm{mm}$ wide continuous metal strip used for electrical connection.

The mesh manufacturing procedure is similar to the one used for making multi-layer printed boards on thin polymer supports, and is only briefly summarized here; a more detailed description will be provided elsewhere [4]. Two identical masks with the desired pattern are realized on plastic film by a computer-assisted laser photo-composer, assembled and optically aligned on a frame holder. The double-side kapton-based circuit, previously coated with a thin film of photo-sensitive resin, is inserted between the masks, and exposed to UV light; after curing, a conventional sequence of solvent and acid baths then exposes and removes the metal from the holes on both sides, followed by an immersion in a kapton-specific solvent, opening cone-shaped channels from both sides. A standard washing and drying procedure completes the fabrication. Best resolutions and definitions of edges have been obtained by gold-plating the copper electrodes before etching the polymer.

Several methods have been tested to prevent the danger of discharges at the ridge on the outer edges of the mesh when applying the potentials. Simple mechanical peeling of a few mm wide strip of copper on one side of the sheet is very effective; a mechanical protection with holding frames of a metal-free strip all around the edges of the mesh, to avoid etching of the underlying kapton, has also been used successfully. Protective coating (passivation) of exposed kapton around the edges with a resin resistant to the solvent would be a better alternative to reduce handling costs, and is being investigated.

After manufacturing, meshes are washed in de-ionized water and dried for a few hours at $80^{\circ} \mathrm{C}$; before assembly, the resistivity in air is checked at $200 \mathrm{~V}$ and yields typical values between a few hundred and a few thousand G $\Omega$. Depending on the application foreseen, the pre-amplifier mesh is mounted on a thin temporary fiberglass support board, few hundred microns thick, or directly on a frame of the type developed for MSGCs [5]. Two copper strips are used to feed the operating potentials. Whenever possible, we have forced the gas circulation through the mesh to improve the uniformity of distribution in the volume of the detector; with its optical transparency between 10 and 20\%, the GEM mesh presents a negligible impedance to the gas flow. All measurements have been realized operating the detectors at atmospheric pressure. 


\section{COUPLING GEM TO A MULTIWIRE PROPORTIONAL CHAMBER}

A GEM mesh has been mounted replacing one cathode plane in a standard small size $\left(10 \times 10 \mathrm{~cm}^{2}\right)$ multi-wire chamber having $5 \mathrm{~mm}$ gaps and anode wires $20 \mu \mathrm{m}$ in diameter and $2.54 \mathrm{~mm}$ spacing; for these measurements, a $200 \mu \mathrm{m}$ pitch mesh (GEM2) was used. For convenience of operation, the lower electrode of GEM as well as the symmetric cathode of the MWPC are grounded, and the pre-amplifying potential is applied to the upper GEM electrode through a high value resistor (Fig. 4). A positive potential is provided to the MWPC anodes, and the drift electrode is independently powered. Signals are read-out from anode wire groups with capacitors; this configuration allows independent operation of GEM and MWPC, as well as the controlled injection of ionization electrons into the pre-amplifier mesh. All measurements have been realized exposing the detector to collimated $5.9 \mathrm{keV}$ X-rays from an external ${ }^{55} \mathrm{Fe}$ source.

An example of recorded pulse height spectra for a moderate voltage applied to GEM, showing both direct and pre-amplified charges, is provided in Fig. 5; the filling gas is argon-dimethylether (DME) in the volume ratio 90-10, and the operating voltages are indicated in the inset. The detected charge as a function of anode voltage, in a range of values for the GEM potential, is shown in Fig. 6; the drift voltage is continuously adjusted to maintain a constant field (about $2 \mathrm{kV} / \mathrm{cm}$ ) in the upper region. We define as pre-amplification (PA) factor the ratio between doubly amplified and direct pulse heights for the $5.9 \mathrm{keV}$ line; for large PA factors, in order not to exceed the dynamic range of the amplifiers and to avoid space-charge distortions, the voltage on the anodes had to be lowered, and the direct signal in the MWPC could not be detected anymore: in this case the factor is deduced from an extrapolation. The highest measured point corresponds to a voltage close to breakdown across the mesh. One should note that, at least in the short term, discharges within GEM are of no consequence to the detector and to the read-out electronics; also, since the leakage current across the mesh is negligible (few nA at $500 \mathrm{~V}$ ), very high value protection resistors can be used. Fig. 7 gives an example of energy resolution of the detector, at a PA factor of 130, for $5.9 \mathrm{keV}$ x-rays; its FWHM $(25 \%)$ corresponds to the one measured on the MWPC alone.

A relevant concern is the efficiency of the collection and transfer process from the upper region into the multiplying channels, hereafter called transparency. Inspection of Fig. 2 reveals that, with typical applied potentials, no field line from the drift region (except for the mathematical boundary between cells) terminates on the upper electrode, thus providing full transmission (transparency equals one). The observed good energy resolution of the pre-amplified signal, generated by photo-electrons having a range comparable with the channels' pitch, confirms that only a small fraction of primary charge, if any, is lost. Increasing the upper drift voltage above the optimum, some field lines reach the electrode, thus gradually reducing the transparency. Lowering the field, on the other hand, results in an increase of diffusion and a loss of electrons by "scraping" the drifting cloud. The width of the transparent region increases with the value of the field in the channel. This behavior is apparent in the measurements of the peak pulse height as a 
function of the drift potential difference $\left(\mathrm{V}_{\mathrm{D}}-\mathrm{V}_{\mathrm{GEM}}\right)$, normalized by the PA factor corresponding to each value of the potentials (see Fig. 8). Similarly, Fig. 9 shows normalized pulse heights measured at constant PA as a function of drift potential, for several voltages applied to the MWPC; in the limited range allowed, the measurement shows the small effect on transferred charge of changing the lower drift field. The measurements merge possible gain changes with modifications in the transfer efficiency, and are improperly called transparency; diffusion of electrons in the multiplying channel, that may lead to loss of charge by collection on the GEM electrodes, may also result in an effective gain smaller that the real avalanche size. These effects will have to be better analyzed both theoretically and experimentally.

The wide tolerance of the operation to the applied external potentials is apparent: inspection of the data shows for example that at a PA factor of around $100\left(\mathrm{~V}_{\mathrm{GEM}}=-360 \mathrm{~V}\right)$ a variation by a factor of five of the drift field results only in a 5\% change in the detected charge; part of the loss is presumably an artifact due to the amplifier response to the changing rise-times of the signal, affected by the value of drift velocity. The tolerance to variations of the lower field is even wider. This implies that the pre-amplification is unaffected by the thickness of the gap, or that the flatness of the mesh plays a modest role in the operation.

We have studied the transparency with the help of the electrostatic program; the issue is complicated by the three-dimensional character of the problem. Preliminary results indicate a general, albeit not accurate, matching to the experimental data. The analysis is continuing, and will include the effects of electron diffusion and of trajectory distortions induced by external magnetic fields (Lorentz angle).

The detector operation has been successfully tested in many gas mixtures, interesting for specific applications where the boost of gain provided by GEM could greatly improve the operating performances. Fig. 10 shows amplification curves measured in helium-methane (70-30), a mixture interesting in view of the use for single electron detection in a large volume Time Projection Chamber for the underground neutrino experiment HELLAZ [6].

The detector works well in argon- $\mathrm{CO}_{2}$, a convenient but gain-limited mixture in conventional gas detectors due to photon-induced secondary cathode emission; aside from separating the overall gain in two smaller and sub-critical steps, the reduced optical transparency of the mesh results in a strong suppression of photon feedback. More results obtained in this nonflammable mixture with a GEM-MSGC will be presented in the next chapter.

A pre-amplification factor around 10 has been also demonstrated in pure DME, despite the very high potential required to operate; this proves also that the intrinsic dielectric rigidity of the grid is determined mainly by gas properties and not by surface breakdown effects. Operation in pure DME is being envisaged for the development of a high resolution vertex Time Projection Chamber for the heavy ions Collider RHIC [7]; combined with another amplifying device, even a modest PA factor as provided by GEM would allow safer operation.

We summarize in Fig. 11 the pre-amplification curves obtained in various gas mixtures tested so far, as a function of the GEM potential. 


\section{GEM AND THE MICRO-STRIP CHAMBER}

\section{1: Experimental set-up}

Coupling a mesh to a multiwire chamber is useful for studying preamplification and transfer properties, and is expected to have applications whenever very large gains are desired (for example in single photo-electron detectors and $\mathrm{RICH}$ counters). More interesting appears the combination with a micro-strip chamber, a device limited to maximum attainable gains of a few thousand by the appearance of discharges [8,9]. We have mounted a GEM mesh with a MSGC plate using the thin frame assembly developed in the framework of the studies for the Compact Muon Solenoid (CMS) tracking detector at CERN $[5,10]$. The gaps MSGC-GEM and GEM-drift electrode are both $3 \mathrm{~mm}$ (Fig. 12); anode and cathode strips are 7 and $100 \mu \mathrm{m}$ wide respectively, at a distance centre to centre of $100 \mu \mathrm{m}$. The plate, manufactured with chromium strips on thin glass having a diamond-like coating with controlled resistivity, has an active area of $100 \times 100 \mathrm{~mm}^{2}$, corresponding to the surface of the mesh. The improved design mesh (GEM3, with $140 \mu \mathrm{m}$ spacing between holes) has been used for this set-up. The anodes in the MSGC can be read-out with fast amplifiers individually or in groups; cathode strips are connected in groups of 20 to the operating voltage through protection resistors. To power the preamplifier mesh, we have used the simple resistive network shown in the figure; the ratio between multiplying and lower drift voltages can be modified replacing the external resistors. The upper drift electrode has an independent power supply: setting this voltage below or above the potential of the upper GEM electrode determines the operating mode (simple or double amplification). The detector was installed in a test set-up including a high rate $X$-ray generator with copper target (main line at $8 \mathrm{keV}$ ), a digitally controlled gas mixing bench and a dedicated on-line data acquisition system; for the discharge studies, an internal alpha emitter $\left({ }^{220} \mathrm{Rn}\right)$ could be added in the gas flow as described in Ref. [9]. Absolute gains have been estimated following the procedure outlined in [11]. Initial measurements have been realized with a standard argon-DME mixture in equal proportions, but a non-flammable mixture of argon with carbon dioxide has also given very satisfactory results.

\section{2: Gain characteristics}

Data in Fig. 13 show the combined amplification power of the structure, operated in a mixture of argon and DME in equal proportions, as a function of cathode voltage in the MSGC and for increasing values of the GEM potential; the upper drift field has been maintained roughly constant throughout the measurements at around $6 \mathrm{kV} / \mathrm{cm}$, whilst the lower drift field varies between 2 and $3 \mathrm{kV} / \mathrm{cm}$, depending on the applied potential $\mathrm{V}_{\mathrm{G}}$. The curve labeled $\mathrm{V}_{\mathrm{GEM}}=0$ corresponds to a pure MSGC operation in detecting ionization released in the lower drift region; all other data represent the charge produced in the upper drift region and pre-amplified before reaching the MSGC. Fig. 14 shows a typical 
pulse height spectrum for direct (lower peaks) and pre-amplified charge, measured on the X-ray generator ( $8 \mathrm{keV}$ and $5 \mathrm{keV}$ argon escape lines).

We have successfully operated the MSGC+GEM detector with a nonflammable gas mixture of argon and carbon dioxide, in the ratio 90-10 (a rather unfavorable one even for conventional wire counters). The result is shown in Fig. 15: while, as expected, the MSGC alone could not be operated safely at gains above few thousand, the combined detector can reach overall gains in excess of $10^{5}$. The advantage of operating a large detector system with non-flammable gases is obvious; a fine tuning of the mixture could be necessary to optimize other parameters (drift velocity, Lorentz angles etc.).

Inspection of Figs. 13 and 15 reveals a fundamental property of the double structure: the maximum voltage that can be applied to the MSGC, limited by discharges, depends very little on the PA factor, implying that effective safe gains more than two orders of magnitude larger can be reached. Alternatively, given the desired gain for a specific application, even a moderate PA factor permits to operate the MSGC at a dramatically reduced voltage, thus reducing or completely eliminating discharge problems (see section 4.5).

Of particular interest is the leftmost set of data in both figures, obtained at the largest value of pre-amplification: a signal with an amplitude corresponding to a gain of several thousand is detected even with no potential applied to the MSGC cathodes. The apparent contradiction with the estimated PA factor (150) is explained by the different properties of signals detected in the avalanche and in the ionization chamber modes. During the avalanche development in the MSGC, most of the charge is produced very close to the anodes; due to the fast time constant of the amplifiers, only a fraction (its fast component) is detected. For long strips, there is moreover an additional process of signal cancellation, due to the capacitive coupling between anodes and cathodes (induced signals are of opposite polarity); we estimate in our conditions the fast anode signal to correspond to about $10 \%$ of the total charge. On the contrary, in absence of multiplication in the MSGC, a charge signal is induced with a rise corresponding to the drift time and an amplitude equal to the total charge $\left(\sim 510^{4}\right.$ electrons for $\left.8 \mathrm{keV} \mathrm{X-rays} \mathrm{and} \mathrm{a} \mathrm{PA} \mathrm{factor} \mathrm{of} \sim 150\right)$. Due to the response of our amplifier (with rise and decay times of $50 \mathrm{~ns}$ ), signals detected in the two modes are very similar. Comparison of the signals on groups of anode and cathode strips in the ionization mode shows also, as expected (Fig. 16), that the cathode-induced charge is identical in sign but about five times larger, roughly corresponding to the ratio of field lines from the drift collected by the two electrodes.

\section{3: Rate capability}

MSGC have been developed and adopted by many experiments mainly because of their very good rate capability, particularly if implemented on controlled resistivity supports (electron-conducting or surface conditioned glass). Efficient operation up to and above rates of $10^{5} \mathrm{~mm}^{-2} \mathrm{~s}^{-1}$ has been demonstrated on MSGC plates of the type used for the present measurements, manufactured on glass coated with a thin diamond-like resistive layer with a 
surface resistivity of $\sim 10^{14} \Omega / \square[12,13]$. We have repeated the rate capability measurement on the combined structure, using the procedures described in the quoted references. The conditions have been chosen to maintain comparable pulse heights in three modes of operation: MSGC only, GEM only and MSGC+GEM. The results, shown in Fig. 17, confirm the high rate capability: the charge gain remains essentially constant within few percent up to the maximum rate we could achieve, around $10^{5}$ counts $/ \mathrm{mm}^{2}$ s, regardless from the mode of operation, thus demonstrating the absence of short term ion-induced charging up or space-charge effects in the GEM channels. One should also note that the fraction of ions receding into and through the GEM channels depends on the applied voltages; in the extreme case of unity gain in the MSGC (GEM amplification only), there are no positive ions produced in the lower gas volume, and, presumably, no substrate charging up and ageing problems.

\section{4: Time-dependent gain shifts}

The presence of an insulator close to the multiplication channels introduces the possibility of dynamic gain shifts due to the deposition of charges and the consequent modification of electric field. Inspection of Fig. 2 shows that no field line connects the multiplication and the drift regions to the insulator, preventing direct charge deposition; collisional diffusion and photon-induced processes can however overcome this intrinsic protection against charging-up.

The early measurements in Ref. [2] did not show gain shifts at moderate rates; the rate capability measurements described in the previous section confirm this observation. In some of the set-ups, however, a moderate shift upwards of the gain has been observed, reaching a constant value with a time constant dependent on the irradiation rate (Fig. 18); once "formed", the gain remains stable as long as the detector is kept under operation. Suspending the high rate irradiation, the gain returns to its original value with a time constant of several hours (Fig. 19): for this measurement, a very low rate $\left(\sim 15 \mathrm{~Hz} \mathrm{~mm} \mathrm{~mm}^{-2}\right)$ has been used. A clear correlation has been established between the extent of gain shift (if any) and the inner diameter of the channels: GEM grids with narrower holes (see Fig. 1b) exhibit charging-up, while those with wide channels do not. We have confirmed that the meshes used for early measurements had relatively larger holes, thus explaining the absence of gain shifts. This point clearly requires further investigation; a better quality control in the manufacturing process is also necessary to obtain the desired geometry.

A simple and effective solution has been found to make the channels slightly conducting, eliminating the charging-up problem: addition of a small controlled amount of water to the gas. The data in Fig. 20 (open circles) show the stability in time of the gain, obtained in the same structure and conditions as for the previous measurement, except for the higher PA factor ( 40), after the addition of $0.35 \%$ of water to an argon-carbon dioxide mixture; a measurements without water is also shown for comparison (full dots). After the addition, the inter-electrode resistivity of the GEM mesh decreases gradually by a factor of 10 (from 100 to $10 \mathrm{G} \Omega$ ) and then remains constant; taking into account the total 
area of the channels, this indicates that a surface resistivity around $10^{16} \Omega / \square$ is sufficient to eliminate charging-up at the highest rates.

It should be mentioned that addition of water has been used for decades in order to prevent ageing processes in gaseous multi-wire detectors; some authors have speculated this to be due to an increased surface conductivity, due to water adsorption, of the thin polymer layers deposited on the electrodes. In the case of the MSGCs, the reduction of dielectric rigidity between anodes and cathodes due to moisture has discouraged this approach [14] but we have verified that the combined GEM-MSGC device can hold safely the reduced potentials required for satisfactory operation, as shown in Fig. 21. It is also conceivable that the reduction in surface resistivity of an insulating MSGC substrate, obtained with water addition, can serve the same purpose as the resistive layer under-coat, thus greatly simplifying the detector construction that could be built on standard untreated glass or polymers; use of thin kapton for the MSGC would also allow two-dimensional read-out. This point requires further investigations. Use of one of the various resistive coating technologies developed for MSGC is being investigated as an alternative solution to prevent charge built-up in the channels; because of the intrinsic non-directionality of the carbon vapor deposition process, the diamond-like coating described in Ref. [13] seems appropriate to obtain the required conductivity.

\section{5: Discharge limits in presence of heavily ionizing tracks}

The tendency of heavily ionizing particles to trigger a discharge in MSGCs has been observed some time ago [15]. Experimental results obtained in real operating conditions have since confirmed that this is a major problem for reliable operation of the detector in a high radiation environment [9]. The process is understood as a spontaneous transition of large avalanches, such as those induced by dense ionization, from the proportional to the streamer regime [16]; a streamer close to the MSGC substrate can then induce a discharge with devastating effects on the strips and on electronics. Various protection schemes have been used, with high value resistors and keeping the interelectrode capacitance as small as possible, but none appears fully satisfactory. Apart from the temporary inhibition of operation after a breakdown, discharges can produce permanent damages to the anodes, particularly when using for the strips low melting point metals such as aluminum or gold [17].

Under the reasonable assumption that the transition probability from avalanche to streamer in the MSGC induced by large ionization densities is voltage dependent, the substantial reduction in the operating potential allowed by the pre-amplification properties of GEM should strongly reduce the discharge rates. To demonstrate this feature we have used the set-up described in Ref. [9] that allows to expose the detector simultaneously to a continuous flux of X-rays and to heavily ionizing $\alpha$ particles resulting from the decay of ${ }^{220} \mathrm{Rn}$ added in the gas flow by a thorium oxide generator ${ }^{2}$. The average energy

\footnotetext{
${ }^{2}$ Following a procedure suggested by B. Schmidt, Physicalishe Inst. Heidelberg, Germany
} 
release in a $3 \mathrm{~mm}$ gap of the $\alpha$ decays is $400 \mathrm{keV}$, emulating the effect of nuclear fragments and neutron conversions in real experiments. With the MSGC alone, we have confirmed our previous observations: at a moderate gain (around 2000, required for detection of minimum ionizing particles) the operation appears safe in the short term under simultaneous exposure to $\sim 10^{4}$ $\mathrm{Hz} / \mathrm{mm}^{2}$ of X-rays and $\sim 5 \mathrm{~Hz} / \mathrm{cm}^{2}$ of $\alpha$ particles. The discharge probability is however different from zero, and the detector trips off (due to its current protection scheme) around once every hour; moreover, the trip-off rate increases with time indicating long-term or irreversible damages in the structure [18].

The result of our measurement with the combined MSGC+GEM structure is simply stated: with a PA factor of $50\left(\mathrm{~V}_{\mathrm{GEM}}=-400 \mathrm{~V}\right)$ and at an overall gain of $5000\left(\mathrm{~V}_{\mathrm{C}}=410 \mathrm{~V}\right)$ no discharge has been detected operating the detector continuously for over 100 hours. Exploiting the modularity of the MSGC output connections, that allow individual grounding of groups of anode strips, we have determined the natural and radiation-induced discharge voltages across the micro-strip sensitive area. As expected, the critical voltage depends from the group (presumably because of imperfections in the manufacturing), and is strongly reduced by the simultaneous presence of a high radiation flux and heavily ionizing particles (Fig. 22). Obviously, the maximum operating voltage of the whole chamber is determined by the worst group. Data for the MSGC alone and for the combined detector, operated for this measurement at a PA factor around 50, do not differ substantially as far as the maximum MSGC operating voltage is concerned; translated however in a scale of effective gain (Fig. 23) the results show the spectacular improvement of the detector, that could be operated safely at amplification factors above $10^{4}$.

Of particular interest in the plots is the point labeled "after spark". During the measurements, the potential on this particular group of anodes was mistakenly increased far above the safe value, resulting in a heavy discharge: after the accident, the potential on this group in the MSGC could not be increased above $450 \mathrm{~V}$. In the combined detector, this is still well above the operating voltage and leaves a comfortable margin of operation.

\section{DISCUSSION OF THE RESULTS AND FUTURE DEVELOPMENTS}

The experimental measurements described in the present work confirm and extend the earlier observations made with the gas electron multiplier device. Large area $\left(\sim 100 \mathrm{~cm}^{2}\right)$ meshes have been successfully operated in conjunction with a conventional multi-wire and a high-rate micro-strip chamber; with $50 \mu \mathrm{m}$ thickness of the polymer between electrodes, gains (preamplification factors) above 100 have been obtained in a wide range of gas fillings, including non-flammable mixtures of argon and carbon dioxide. A wide tolerance for correct performance to the value of the external fields makes the GEM grids very effective and insensitive to mechanical imperfections in the detector construction; virtually self-supporting, the mesh can be kept in position by simple gluing on thin frames, in good match with the light 
assembly schemes developed for low-mass detectors. When coupled to a MSGC, the additional gain provided by the GEM mesh permits the operation of the combined detector at substantially reduced voltages thus largely increasing its reliability, particularly in harsh conditions of use; safe operation has been demonstrated in non-flammable, poorly quenched gases and under the simultaneous exposure to a large flux of radiation and to heavily ionizing tracks.

A tendency of GEM grids having particularly narrow holes in the center of the insulator to dynamically charge up, with a consequent small increase in gain (reaching a constant value) has been observed. This effect, not very serious by itself (due to the very large dynamics of gain permitted by the use of GEM) can be avoided optimizing the etching procedure in order to obtain larger channel widths. Alternatively, a simple and effective way to avoid charging-up has been found: addition of a small percentage of water $(0.3-0.5 \%)$ to the gas mixture; the addition reduces the surface resistivity in the insulating channels to around $10^{16} \Omega / \square$. While other methods for controlling the resistivity are known and will be investigated, we speculate that water addition can have two other beneficial effects: reduce ageing and naturally provide the surface resistivity required to a bare boro-silicate glass or polymer support for high rate applications. Correct operation of the combined MSGC+GEM detector has been verified in the moisturized gas.

A peculiar and interesting mode of operation has been discovered: large signals are obtained with the last detection element maintained at unity gain (ionization mode). This opens up the possibility of eliminating the MWPC or MSGC as final multiplier, and replacing it with a much simpler, cheaper and more reliable stripped printed circuit. The advantages offered by this mode of operation are many: the readout circuit can be patterned in any desired geometry (strips, rings, pads...) depending on application; use of electrically semi-transparent (narrow) strips on a thin insulating support permits the pickup of a controlled fraction of the signal on electrodes on the back side, in order to obtain two-dimensional read-out; absence of ions produced in the last gas volume eliminates problems of support charging-up and, possibly, of ageing.

Medium and long term behavior of the GEM detectors remain to be tested, as well as their localization properties, efficiency plateau and operation in magnetic field. Alternative methods of fabrication, as well as the use of lighter metals for the electrodes are also to be investigated.

\section{ACKNOWLEDGMENTS}

This is the last paper susceptible of being quoted in further works as "Bouclier et al.", following Roger Bouclier honorable retirement. His precious contribution to more than 35 years of activity as Georges Charpak's technician, and more recently within the Gas Detector Development group is here recognized.

This work would not have been possible without the enthusiastic and competent help of CERN's Surface Treatment group (R. De Oliveira, A. Gandi 
and L. Mastrostefano) in refining the GEM manufacturing technology; their essential contribution to the present work is here acknowledged. 


\section{REFERENCES}

[1] F. Sauli, To be published in Nucl. Instrum. Methods in Phys. Res. (1997) .

[2] R. Bouclier, M. Capeáns, W. Dominik, M. Hoch, J.-C. Labbé, G. Million, L. Ropelewski, F. Sauli, and A. Sharma, The Gas Electron Multiplier, IEEE Nucl. Sci. Symposium (Anaheim, 1997). CERN-PPE/96-177.

[3] A. Sharma and F. Sauli, Nucl. Instrum. Methods A323 (1992) 280.

[4] A. Gandi, L. Mastrostefano, and R.D. Oliveira, Manufacturing procedures for the Gas Electron Multiplier, in preparation at CERN (1997).

[5] R. Bouclier, M. Capeáns, C. Garabatos, G. Manzin, G. Million, L. Ropelewski, F. Sauli, L. Shekhtman, T. Temmel, and G. Fischer, Nucl. Instrum. Methods A367 (1995) 163.

[6] G. Laurenti, S. Tzamarias, G. Bonvicini, A. Zichichi, J. Seguinot, and T. Ypsilantis, CERN/LAA/PC/93-10 (1993).

[7] H. Wieman, Personal communication at Lawrence Berkeley Lab. (1996).

[8] R. Bouclier, M. Capeáns, A. Di Mauro, M. Hoch, L. Ropelewski, F. Sauli, A. Sharma, and L. Shekhtman, Gain limits in different types of microstrip gas chambers with several gases in presence of strongly ionizing radiation, CERN CMS-TN/96-018 (1996).

[9] B. Boimska, R. Bouclier, M. Capeáns, S. Claes, W. Dominik, M. Hoch, G. Million, L. Ropelewski, F. Sauli, A. Sharma, L. Shekhtman, W. VanDonink, and L. VanLanker, Study of ageing and gain limits of Microstrip Gas Chambers at high rates, 5th Int. Conf. on Advanced Technology and Particle Physics (Como, 7-11 October, 1996).

[10] R. Bouclier, M. Capeáns, C. Garabatos, G. Manzin, G. Million, L. Ropelewski, F. Sauli, L.I. Shekhtman, T. Temmel, G. Della Mea, G. Maggioni, and V. Rigato, Nucl. Instrum. Methods A367 (1995) 168.

[11] J. Bohm, R. Bouclier, M. Capeans, C. Garabatos, G. Manzin, G. Million, F. Sauli, T. Temmel, and L. Shekhtman, Nucl. Instrum. Methods A360 (1995) 34.

[12] R. Bouclier, M. Capeáns, M. Hich, G. Million, L. Ropelewski, F. Sauli, and T. Temmel-Ropelewski, IEEE Trans. Nuc. Sci. NS-43 (1996) 1220.

[13] R. Bouclier, M. Capeáns, G. Million, L. Ropelewski, F. Sauli, T. Temmel, R.A. Cooke, S. Donnel, S.A. Sastri, and N. Sonderer, Nucl. Instrum. Methods A 369 (1996) 328.

[14] R. Bouclier, M. Capeáns, C. Garabatos, G. Manzin, G. Million, L. Ropelewski, F. Sauli, L. Shekhtman, K. Silander, and T. Temmel-Ropelewski, Nucl. Instrum. Methods A381 (1996) 289.

[15] R. Bouclier, M. Capeáns, C. Garabatos, G. Manzin, G. Million, L. Ropelewski, F. Sauli, T. Temmel, L. Shekhtman, V. Nagaslaev, Y. Pestov, and A. Kuleshov, Nucl. Instrum. Methods A365 (1995) 65.

[16] V. Peskov, B.D. Ramsey, and P. Fonte, Int. Conf. on Position Sensitive Detectors (Manchester, 9-13 September 1996) LIP/96-9.

[17] H.S. Cho, W.S. Hong, N. Palaio, J. Kadyk, K.B. Luk, V. Perez-Mendez, K.S. Joo and J. Vujic, Spark damage studies of Microgap gas chambers with varipus anode strip metals, 5th Int. Conf. on Advanced Technology and Particle Physics (Como, 7-11 October, 1996).

[18] B. Boimska, R. Bouclier, M. Capeáns, W. Dominik, M. Hoch, G. Million, L. Ropelewski, F. Sauli and A. Sharma, Investigation of discharge limits in diamond coated microstrip gas chambers, CERN-CMS-Tracking Note1996/016(1996). 


\section{FIGURECAPTIONS}

Fig. 1: Micro-photographs of the GEM3 mesh (a); channels are conical in shape, $\sim 100 \mu \mathrm{m}$ wide at the metal level at a pitch of $140 \mu \mathrm{m}$, and 40 to $80 \mu \mathrm{m}$ in the middle of the insulator, depending on the duration of etching (b).

Fig. 2: Equipotentials and field lines computed for a GEM mesh having $50 \mu \mathrm{m}$ insulator and $18 \mu \mathrm{m}$ conductors; the difference of potential applied across the mesh is $360 \mathrm{~V}$, and the drift fields above and below $4 \mathrm{kVcm}^{-1}$.

Fig. 3: Field strength along a central line through the channel.

Fig. 4: Schematics of the MWPC+GEM assembly.

Fig. 5: Direct (lower peaks) and pre-amplified ${ }^{55} \mathrm{Fe}$ signals in the MWPC+GEM structure, recorded at a moderate pre-amplification factor.

Fig. 6: Peak pulse height as a function of the anode potential in the MWPC, for several values of the GEM voltage (gas mixture argon-DME 90-10).

Fig. 7: Example of pulse height resolution for ${ }^{55} \mathrm{Fe} X$-rays at a pre-amplification factor of 130 (argon-DME 90-10).

Fig. 8: Normalized pulse height (transparency) of the GEM mesh as a function of potential in the upper drift region, for several GEM voltages.

Fig. 9: Transparency as a function of drift potential, for fixed GEM potential and several MWPC voltages (normalized to unity gain).

Fig. 10: Amplification curves in a 70-30 helium-methane mixture.

Fig. 11: Pre-amplification factors measured in various gas mixtures, as a function of the voltage across the electrodes of GEM.

Fig. 12: Schematics of the MSGC+GEM assembly.

Fig. 13: Combined absolute gain of the MSGC+GEM detector, as a function of cathode voltage in the MSGC and potential across the GEM mesh. Gas filling: argon-DME (50-50).

Fig. 14: Direct (lower peaks) and pre-amplified spectra in the MSGC+GEM detector for $8 \mathrm{keV}$ X-rays.

Fig. 15: Operation of the combined MSGC+GEM in a non-flammable gas mixture of argon and carbon dioxide (90-10).

Fig. 16: Signal detected in the ionization mode on a group of 16 anode strips (lower track, $20 \mathrm{mV} /$ div.) and of 20 cathode strips (upper track, $100 \mathrm{mV} / \mathrm{div}$.).

Fig. 17: Rate capability (gain as a function of flux) measured with the MSGC+GEM detector in various settings and using the $8 \mathrm{keV}$ X-ray generator.

Fig. 18: Time evolution of gain from power on of a GEM mesh with narrow $(\sim 60 \mu \mathrm{m})$ holes in the center of the kapton foil, measured at increasing rates. Gas filling argon- $\mathrm{CO}_{2}(72-28)$.

Fig. 19: Charging up at high rate and recovery in absence of radiation of a GEM exhibiting gain instability.

Fig. 20: Stability of gain of the GEM mesh at high rate after the addition of a small percentage $(0.35 \%)$ of water to the gas mixture (full dots). A previously obtained curve, measured at a similar rate before the water addition, is show for comparison (open dots). Pre-amplification factor $\sim 40$.

Fig. 21: Operation of the MSGC+GEM detector in a mixture of argon- $\mathrm{CO}_{2}$ with the adition of $0.35 \%$ of water.

Fig. 22: Maximum voltage before discharge on 32 anode groups in the MSGC, in various conditions of irradiation and gain sharing between MSGC and GEM.

Fig. 23: Maximum total gain before discharge in the MSGC, in various conditions of irradiation and gain sharing between MSGC and GEM. 


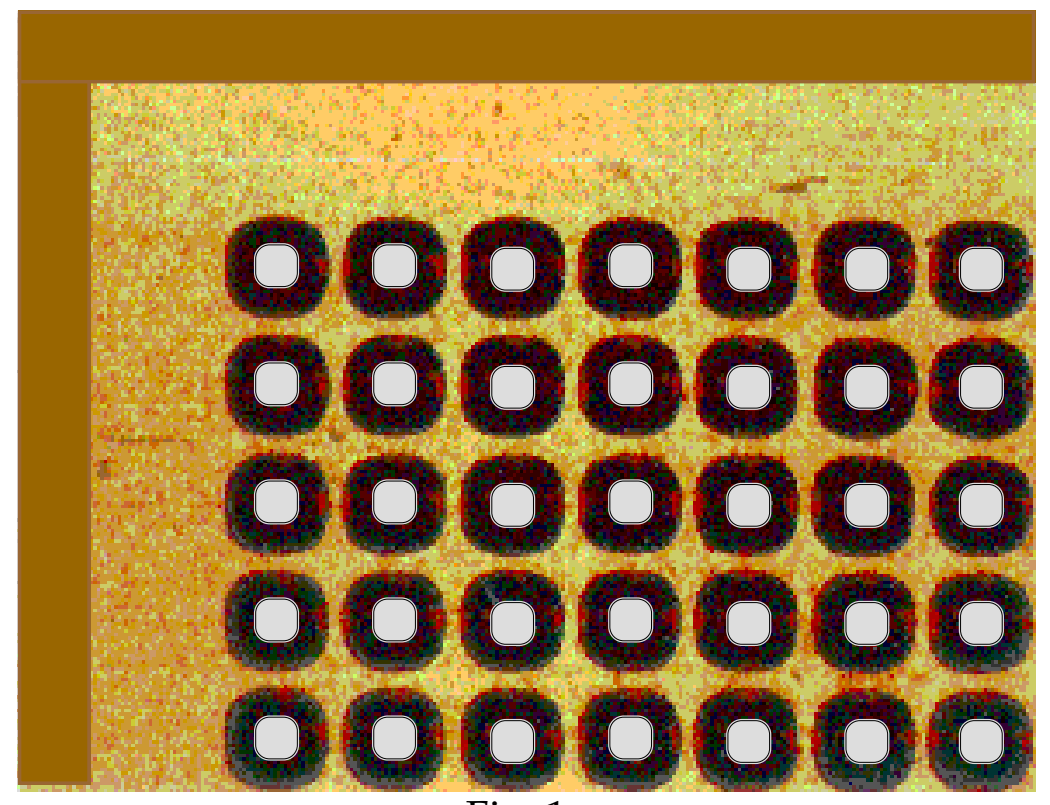

Fig. 1a
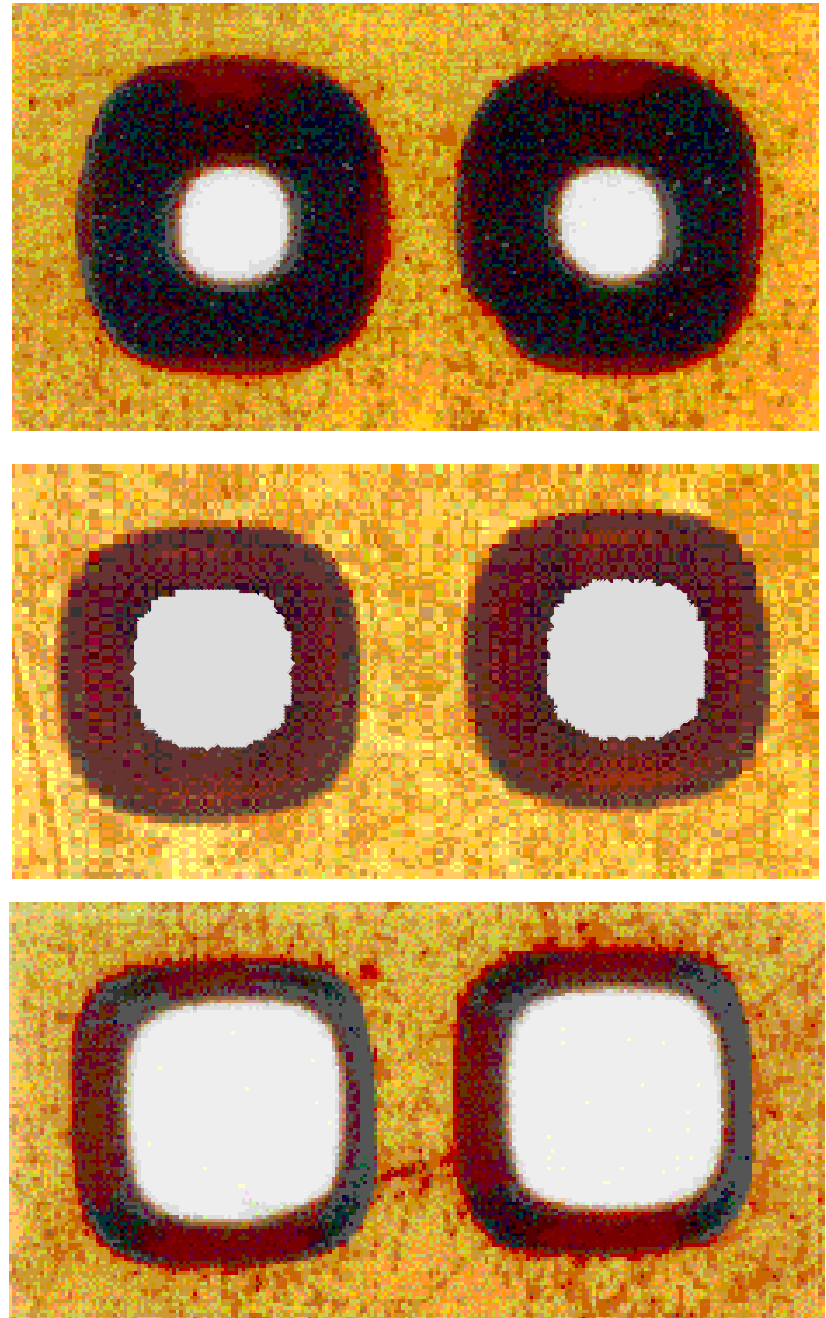

Fig. 1 b 


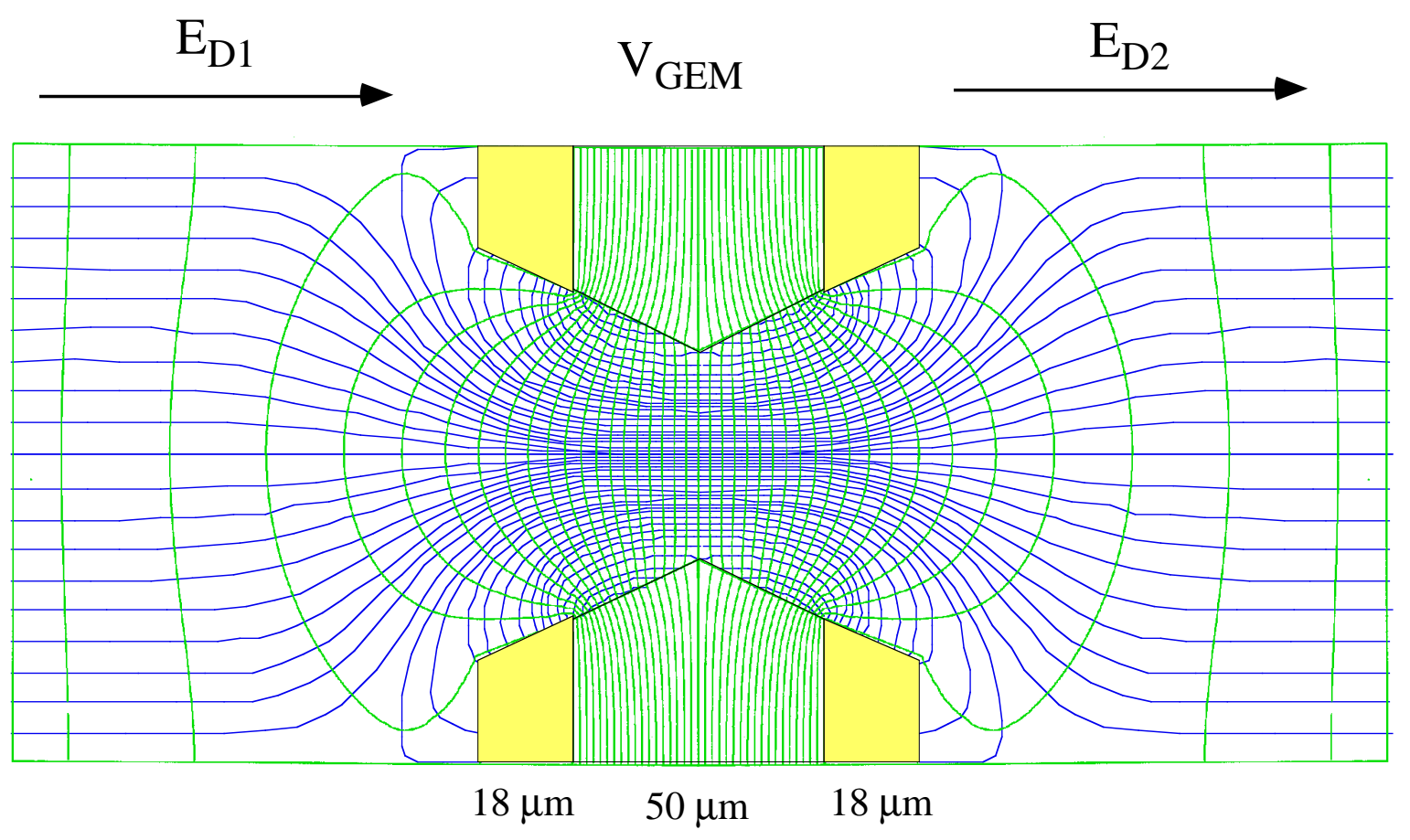

Fig. 2

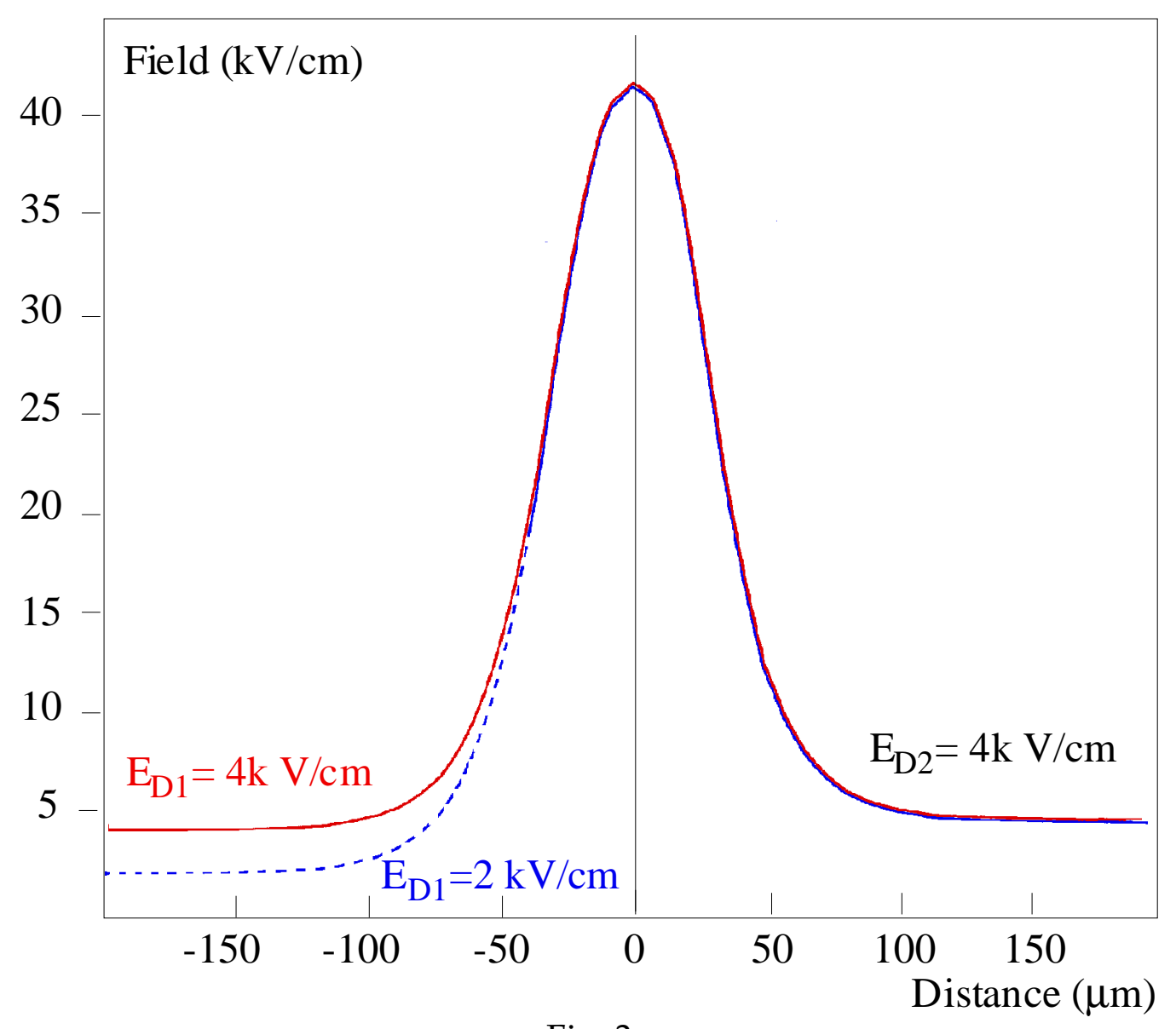

Fig. 3 


$$
\mathbf{v}_{\mathbf{D}} \cdot \mathrm{MM}^{\mathrm{L}}
$$
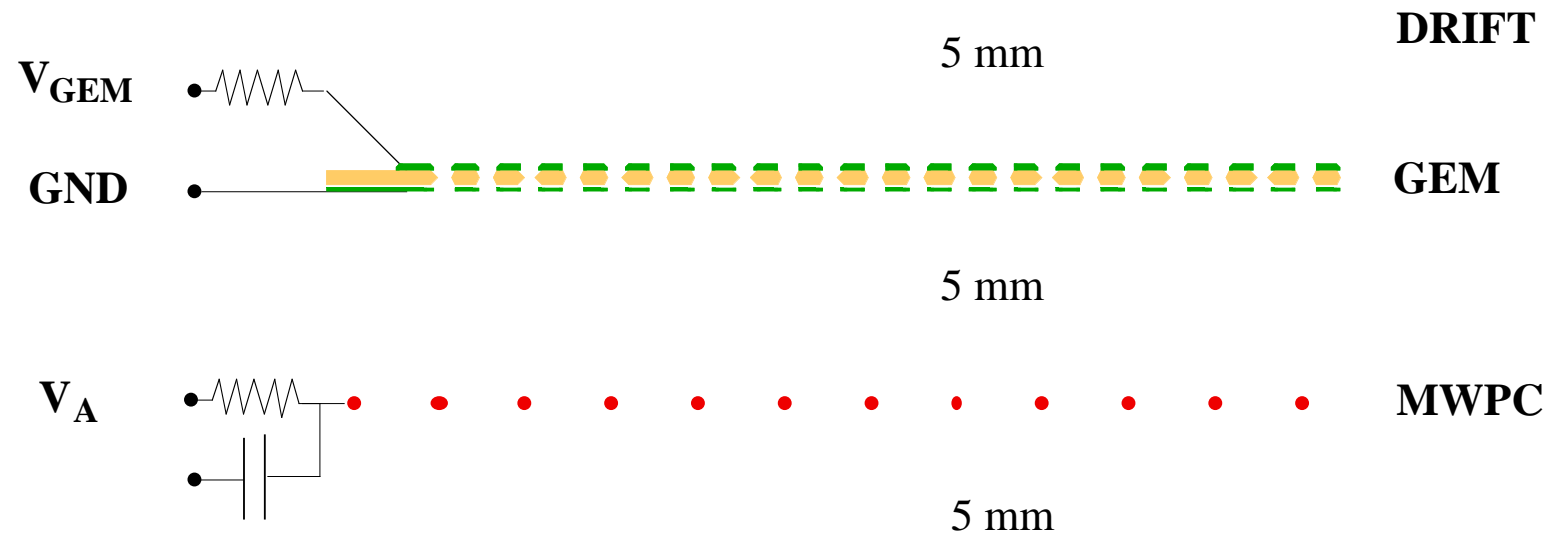
GND

Fig. 4

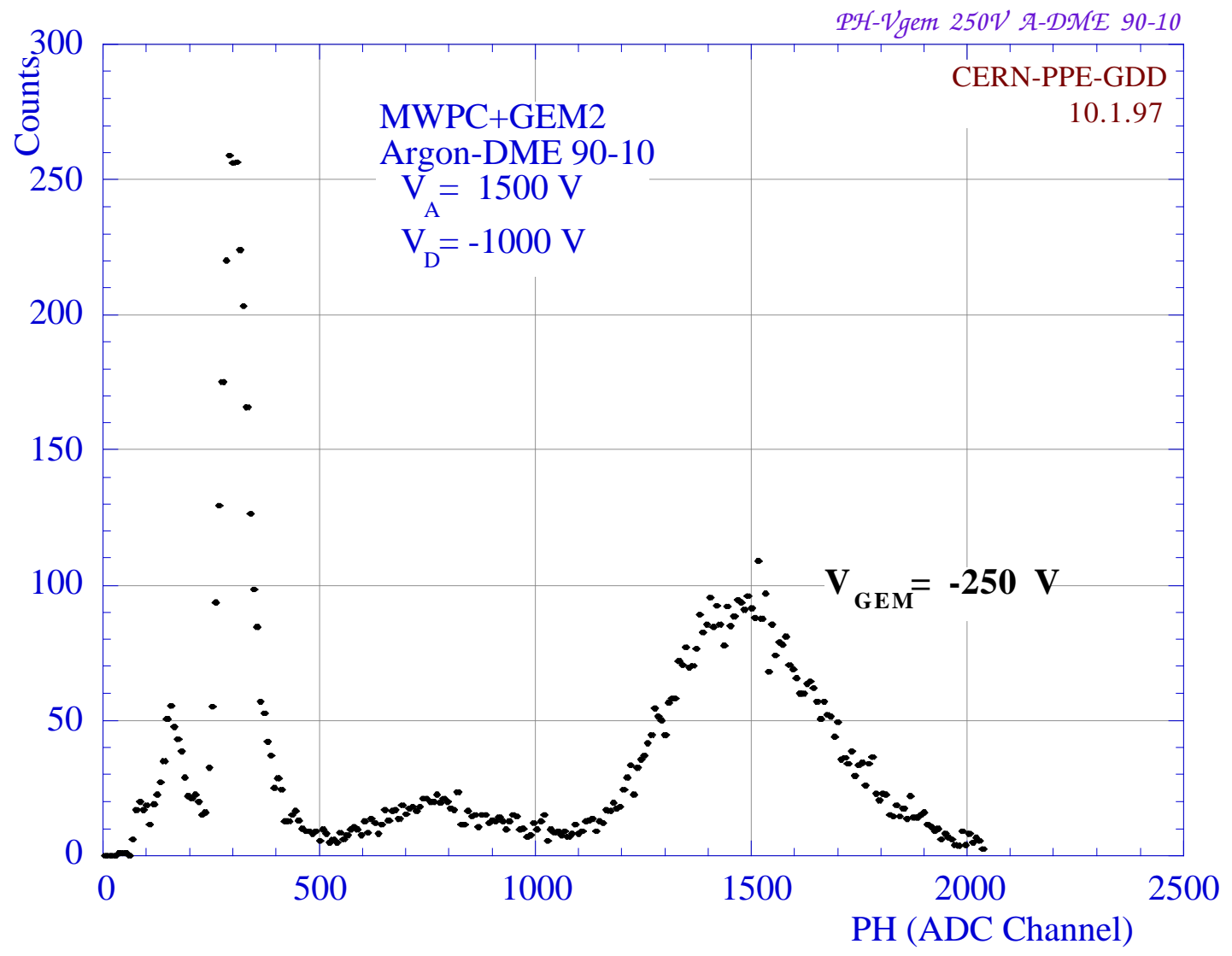

Fig. 5 


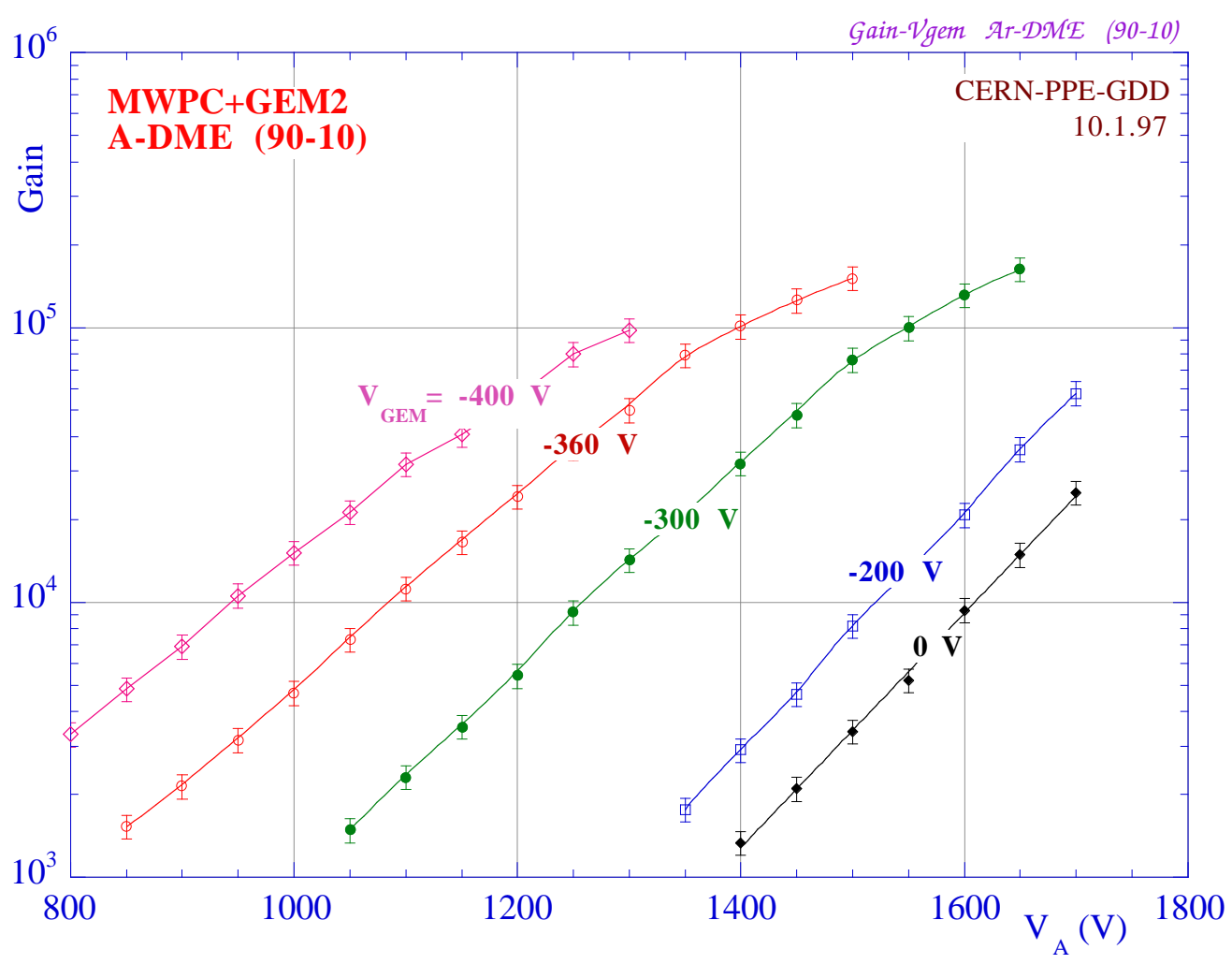

Fig. 6

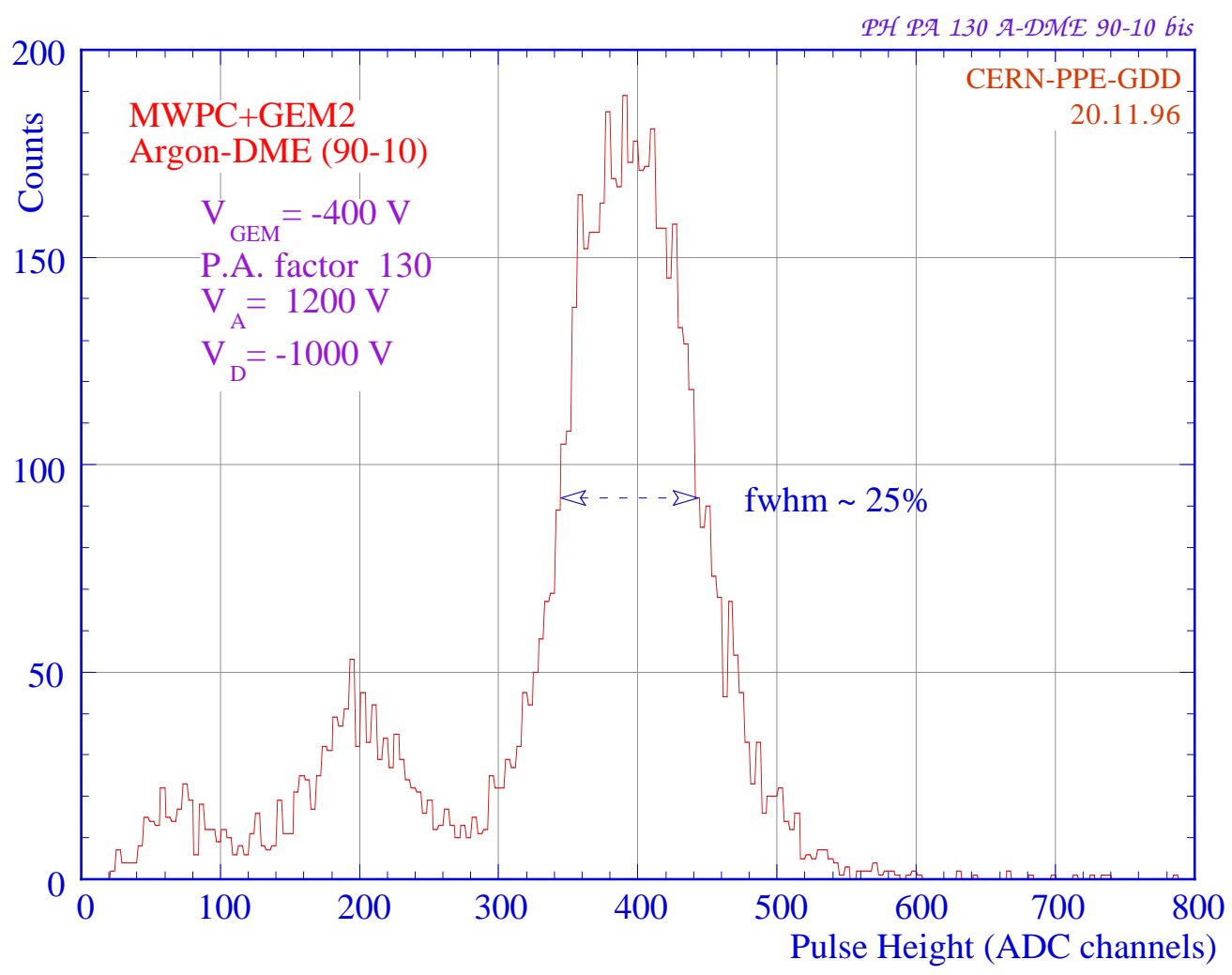

Fig. 7 


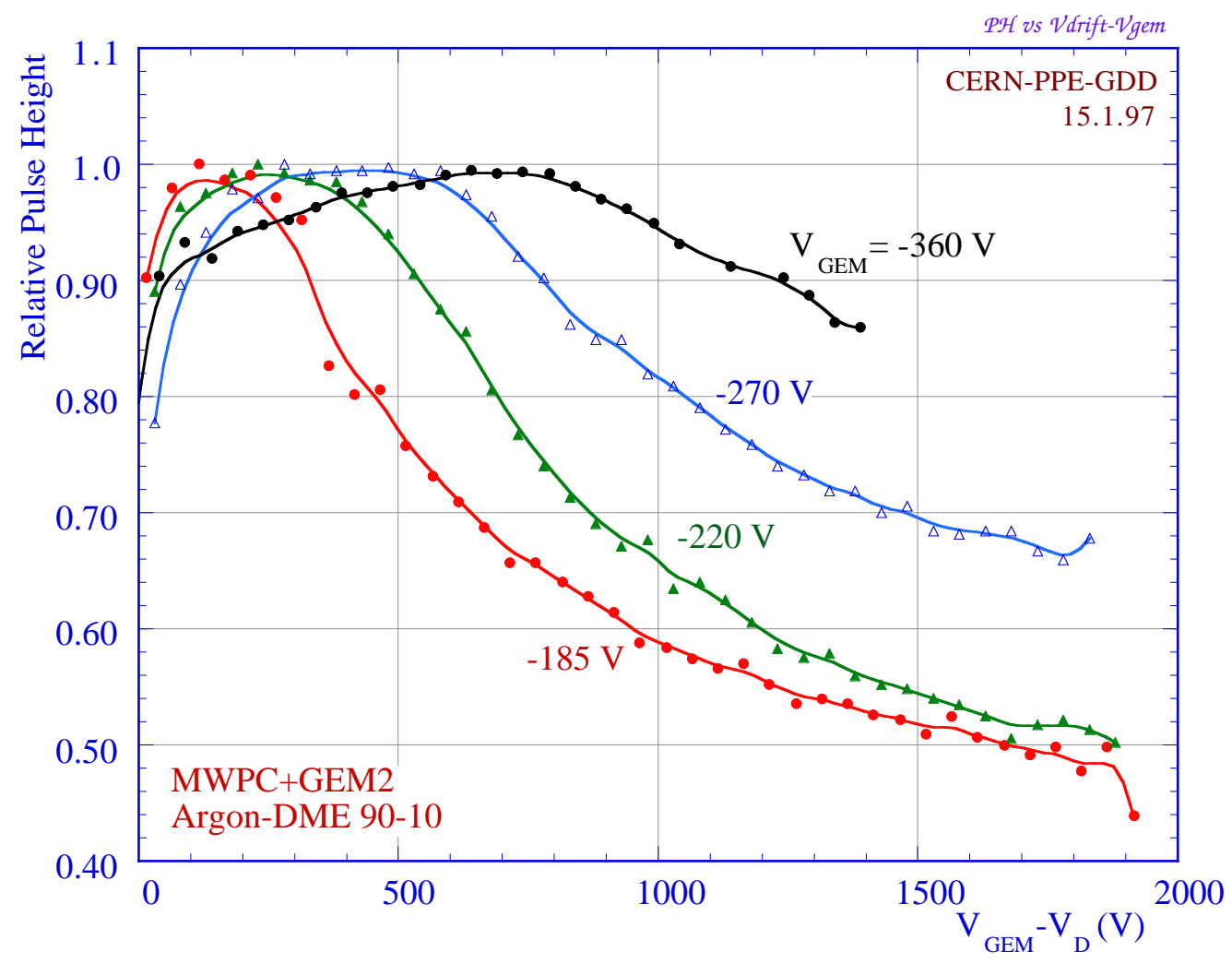

Fig. 8

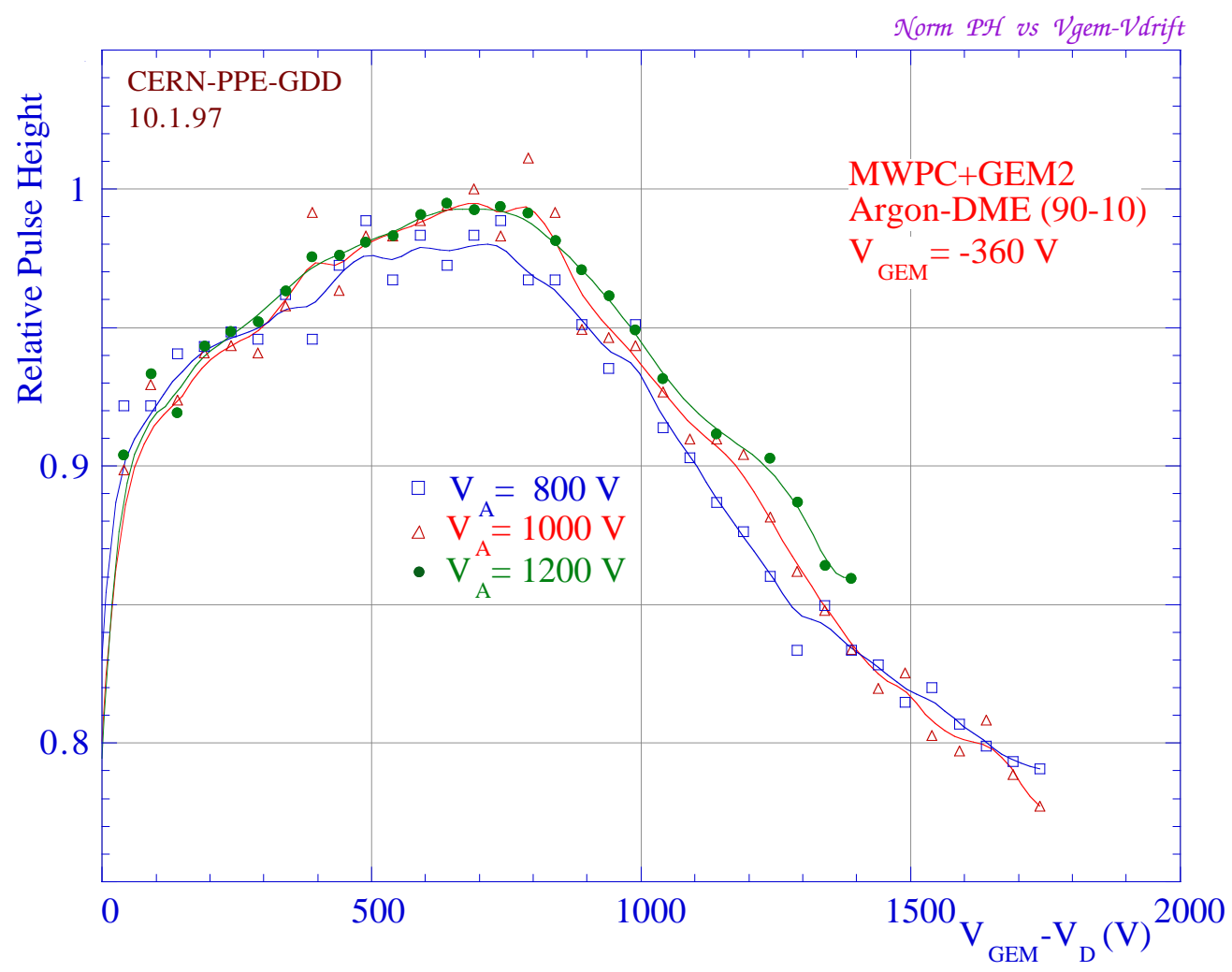

Fig. 9 


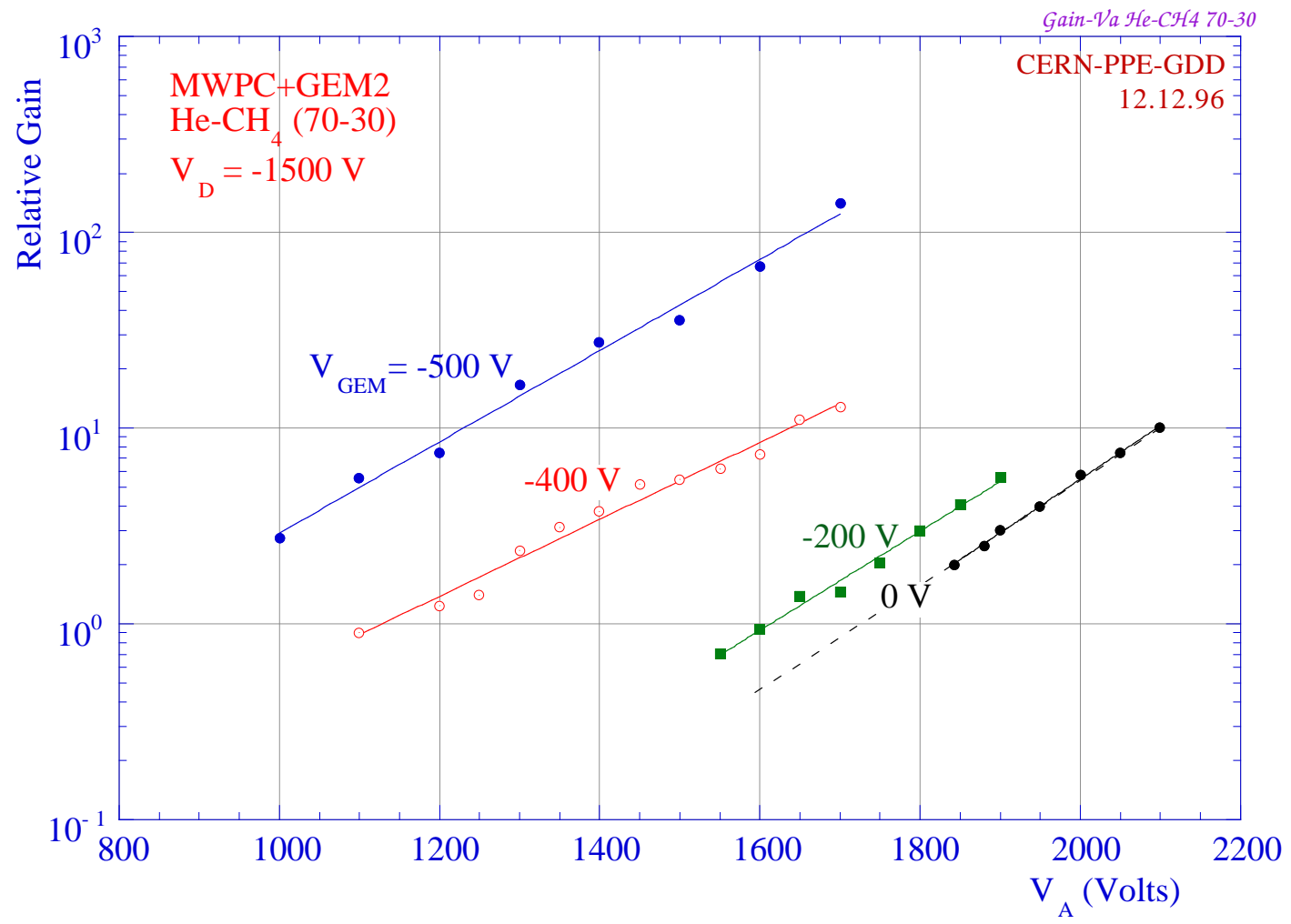

Fig. 10

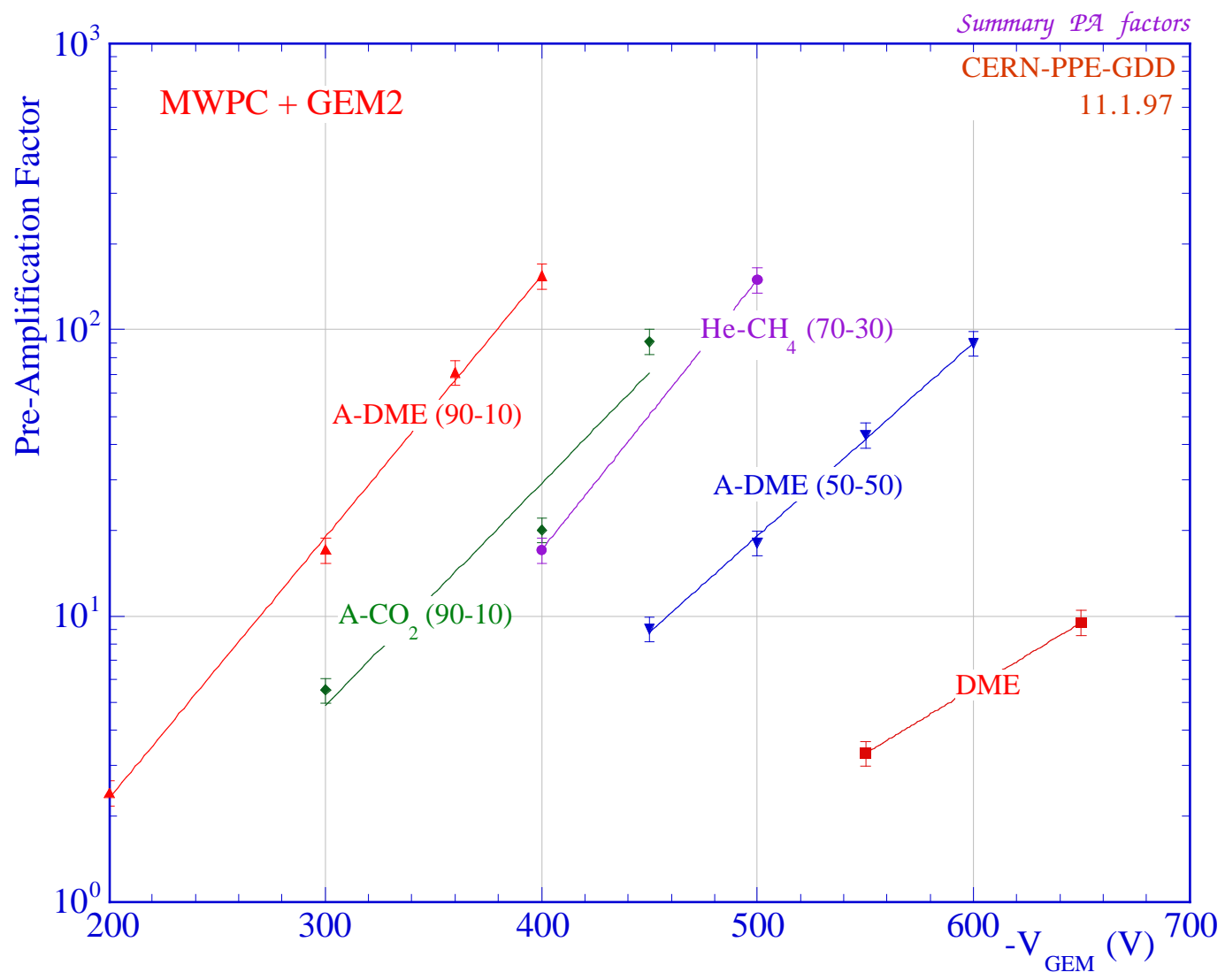

Fig. 11 
$\mathbf{v}_{\mathbf{D}} \cdot W W$

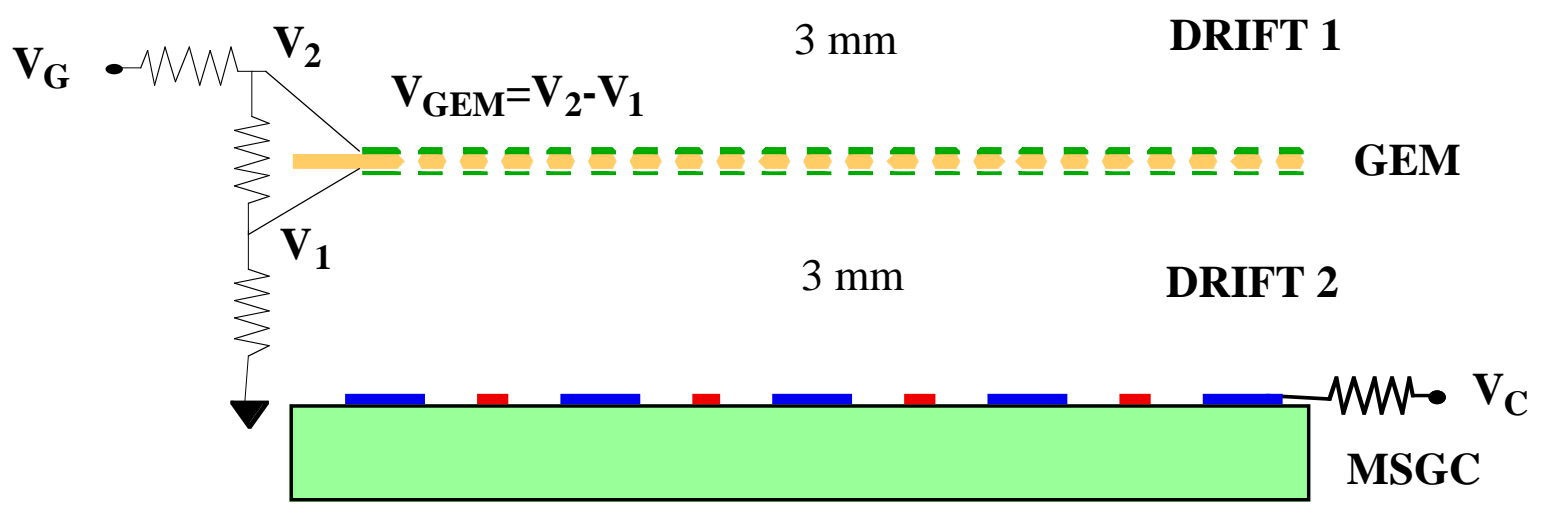

Fig. 12

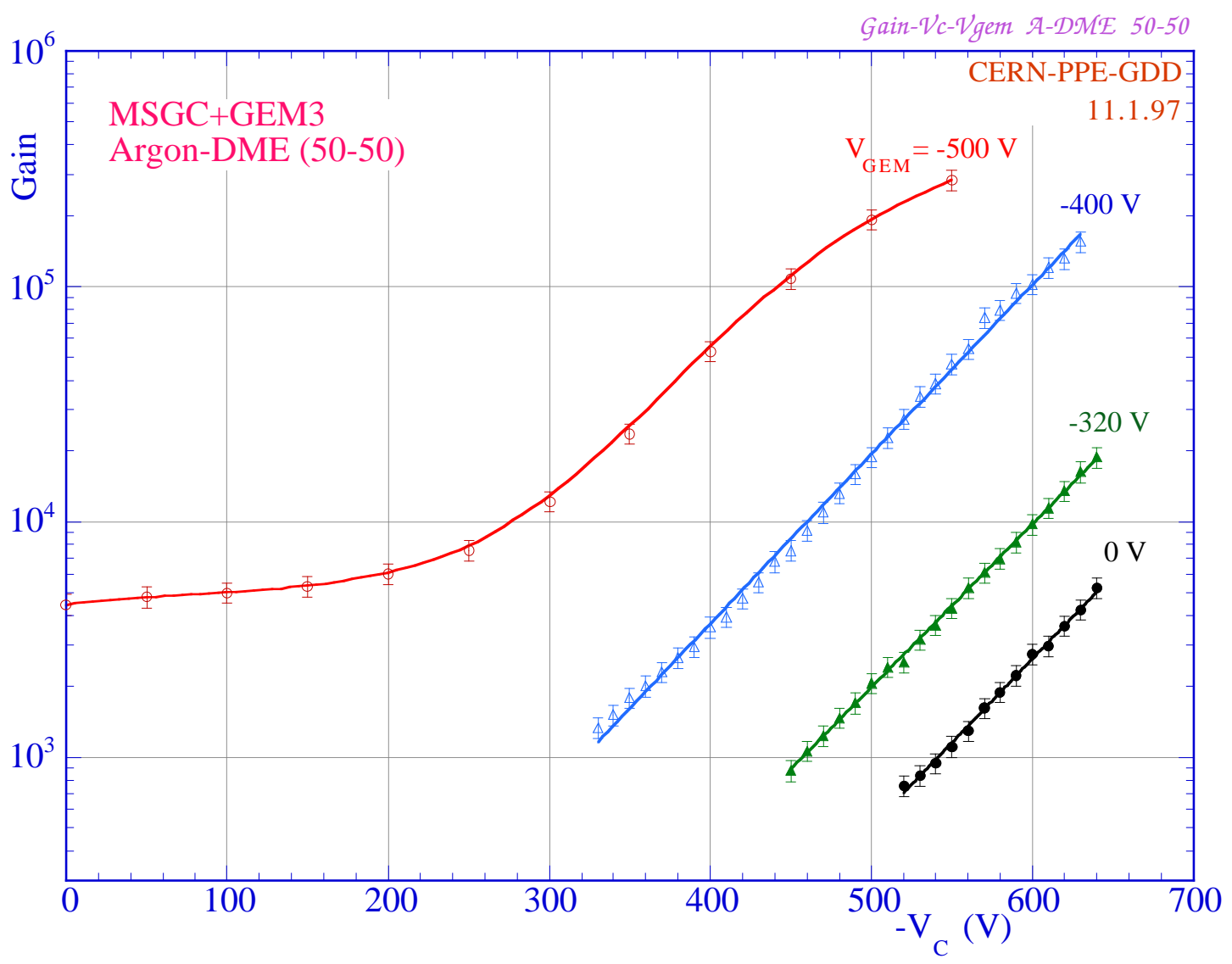

Fig. 13 


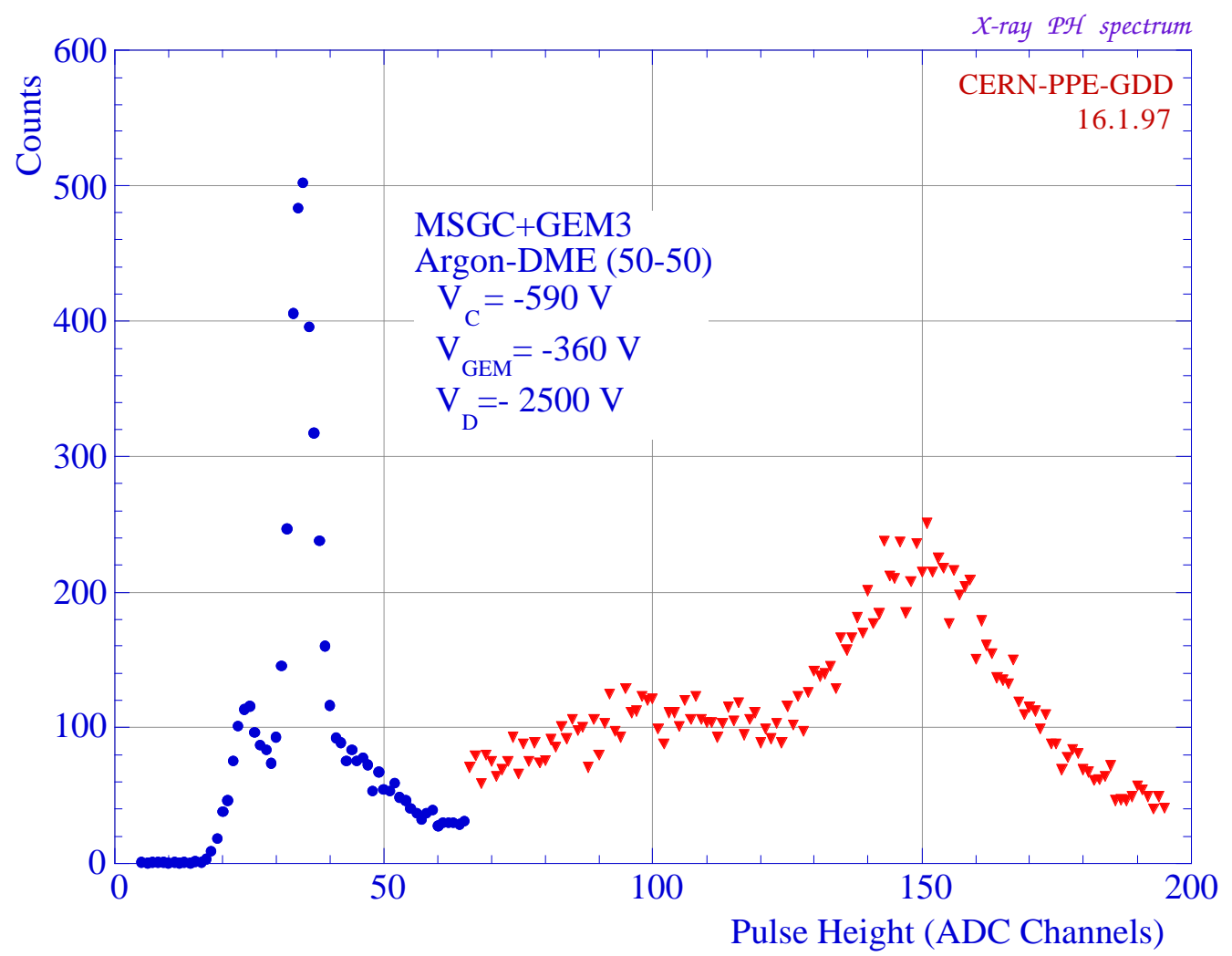

Fig. 14

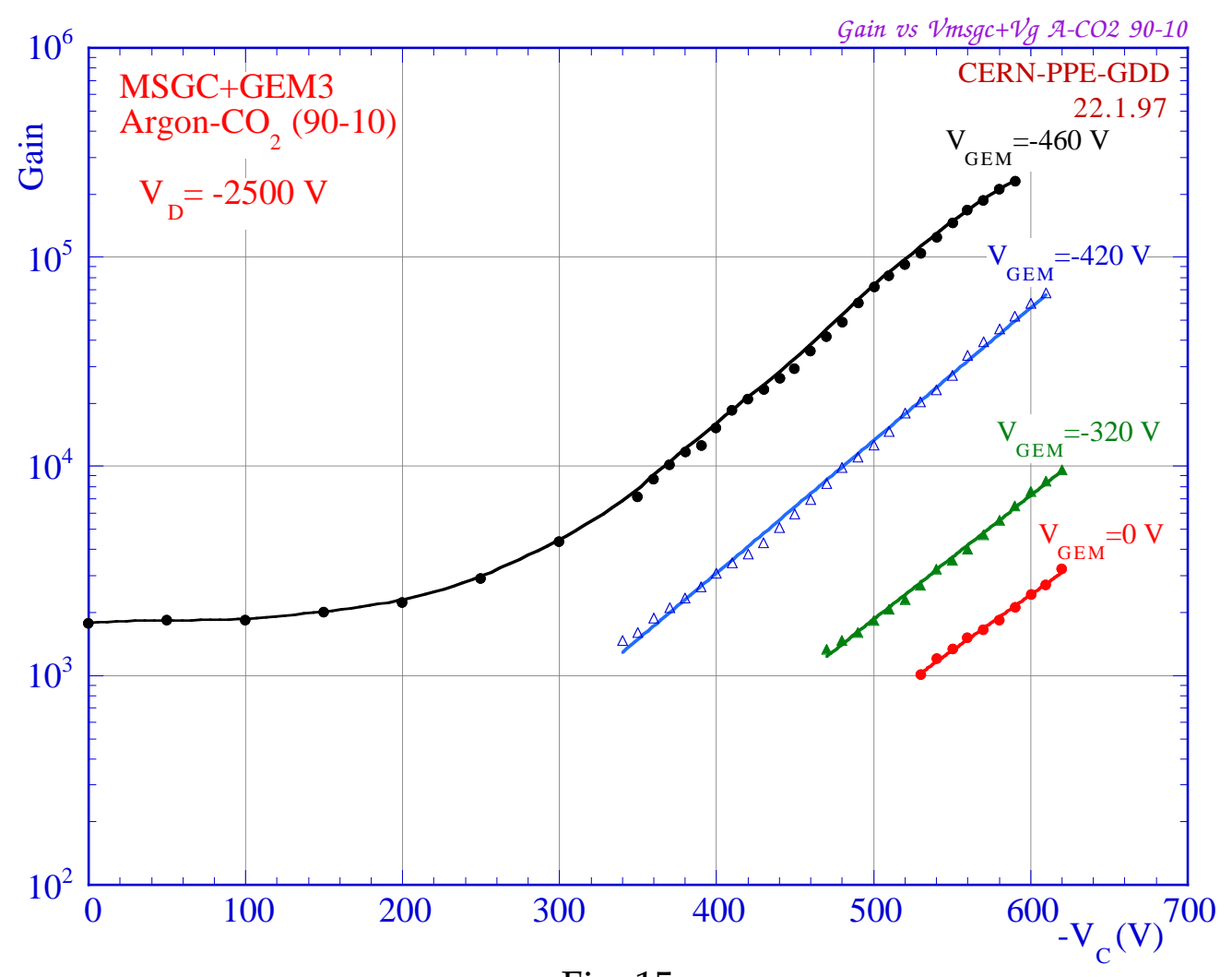

Fig. 15 


\section{A1 $-0.035 U$}

$100 \mathrm{mV} \quad 20 \mathrm{mV}$

Fig. 16

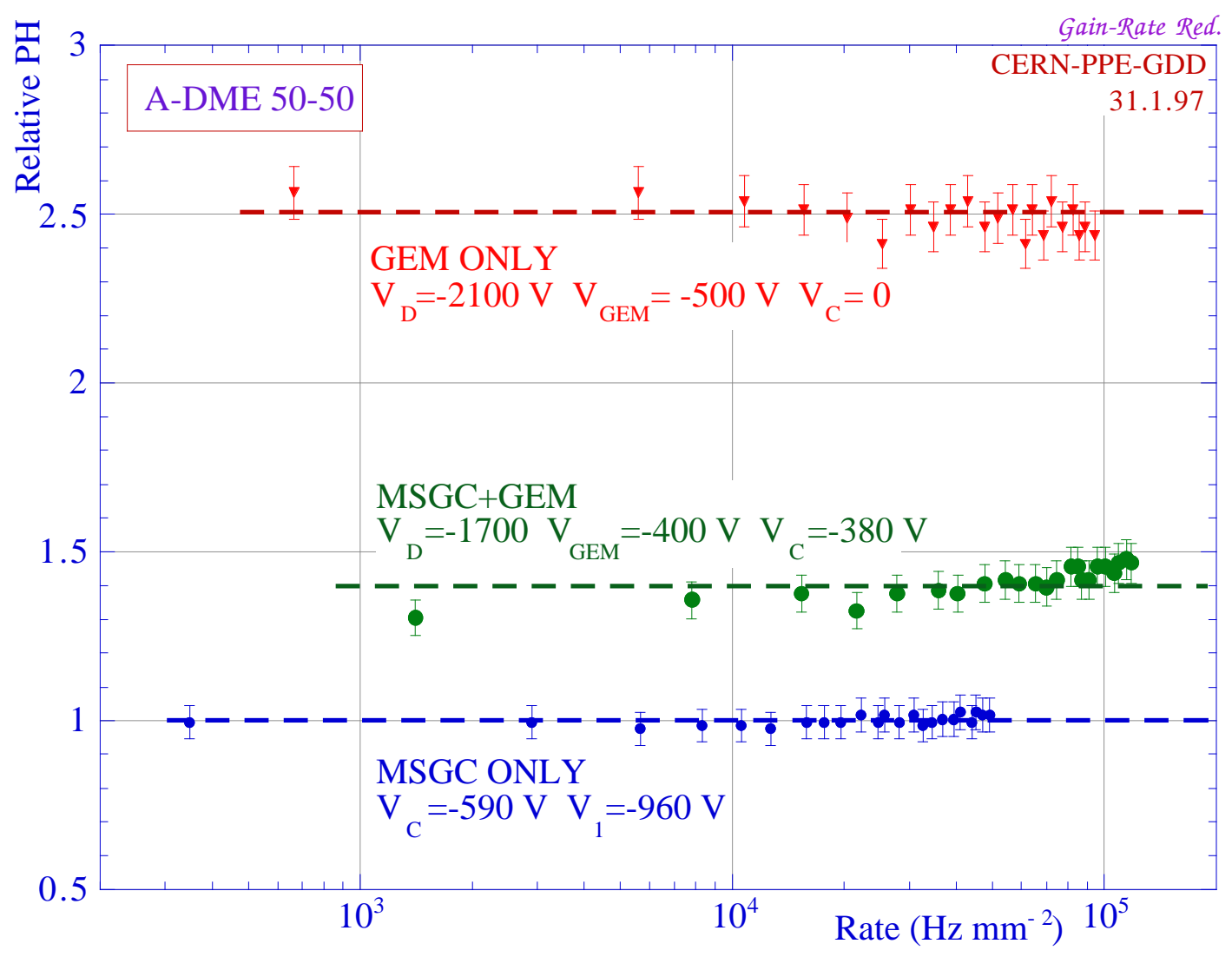

Fig. 17 


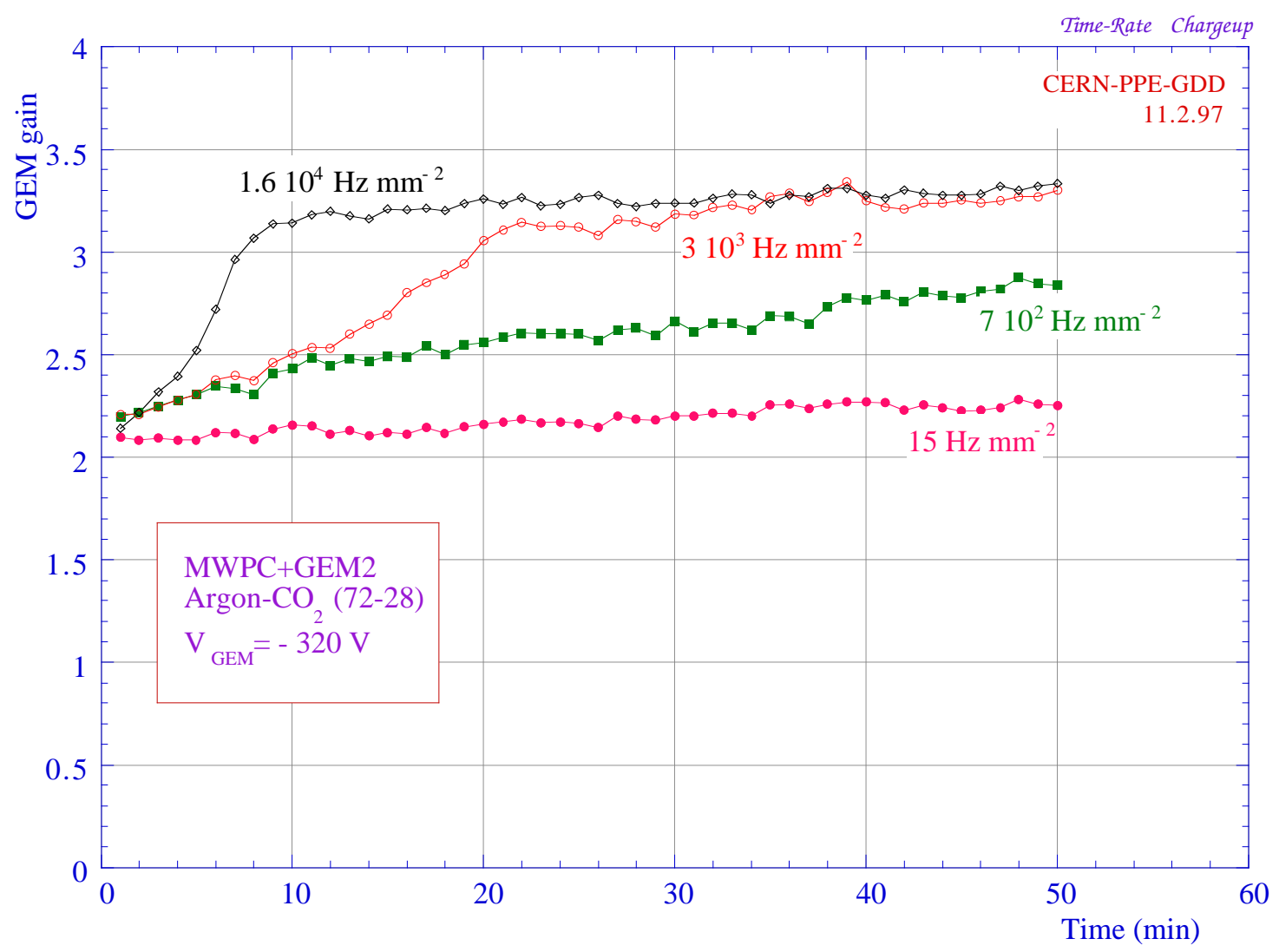

Fig. 18

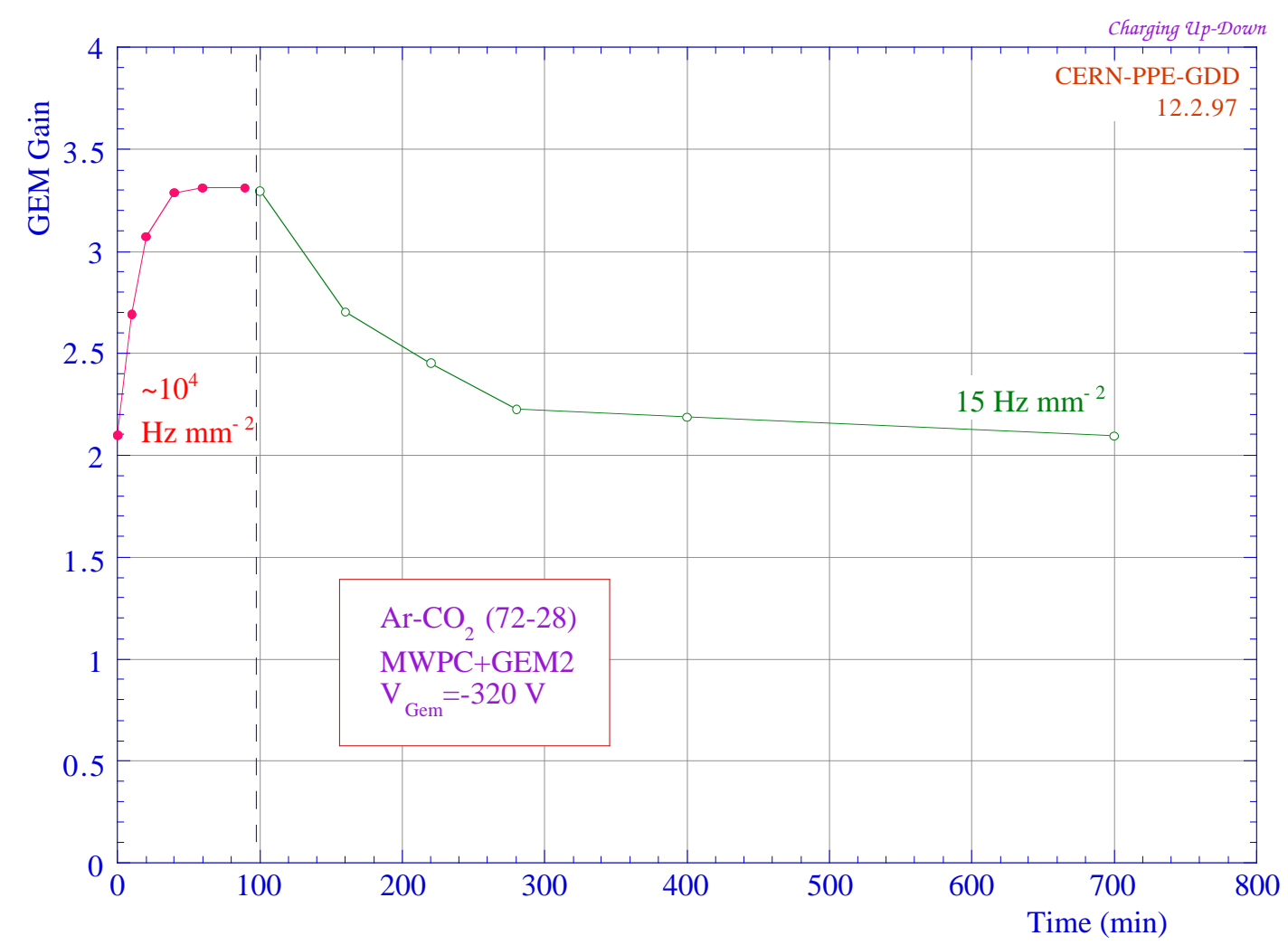

Fig. 19 


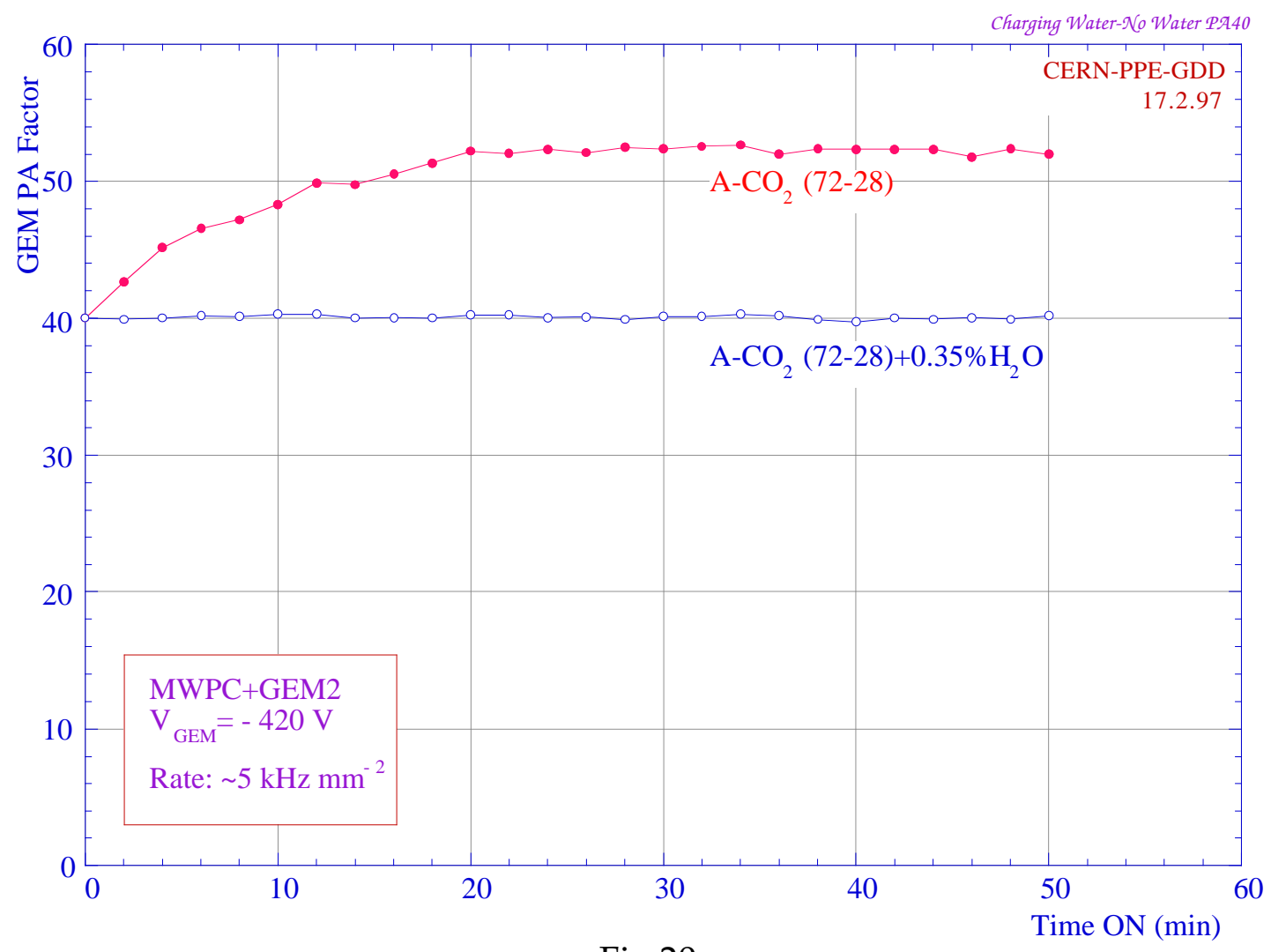

Fig.20

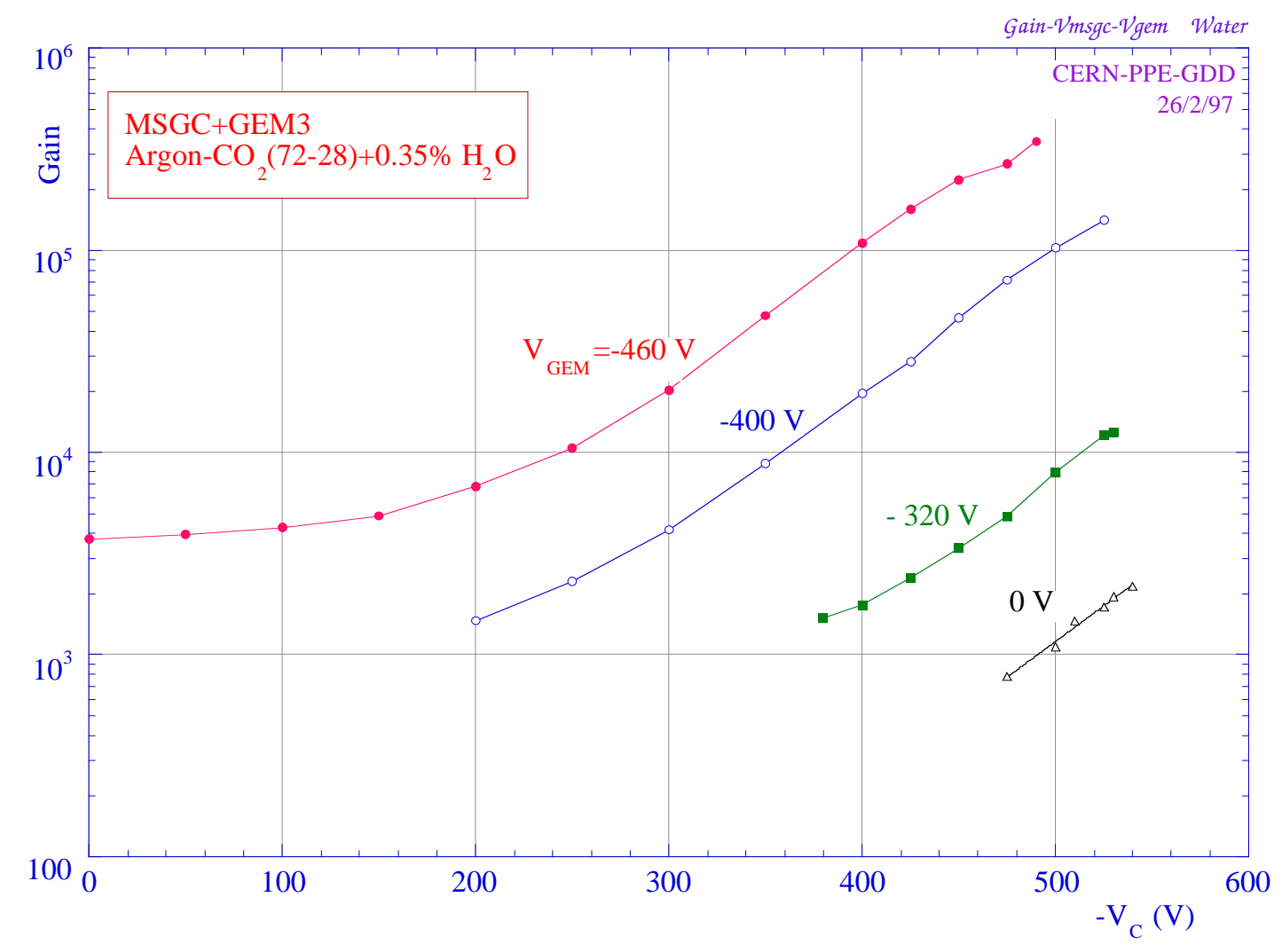

Fig. 21 


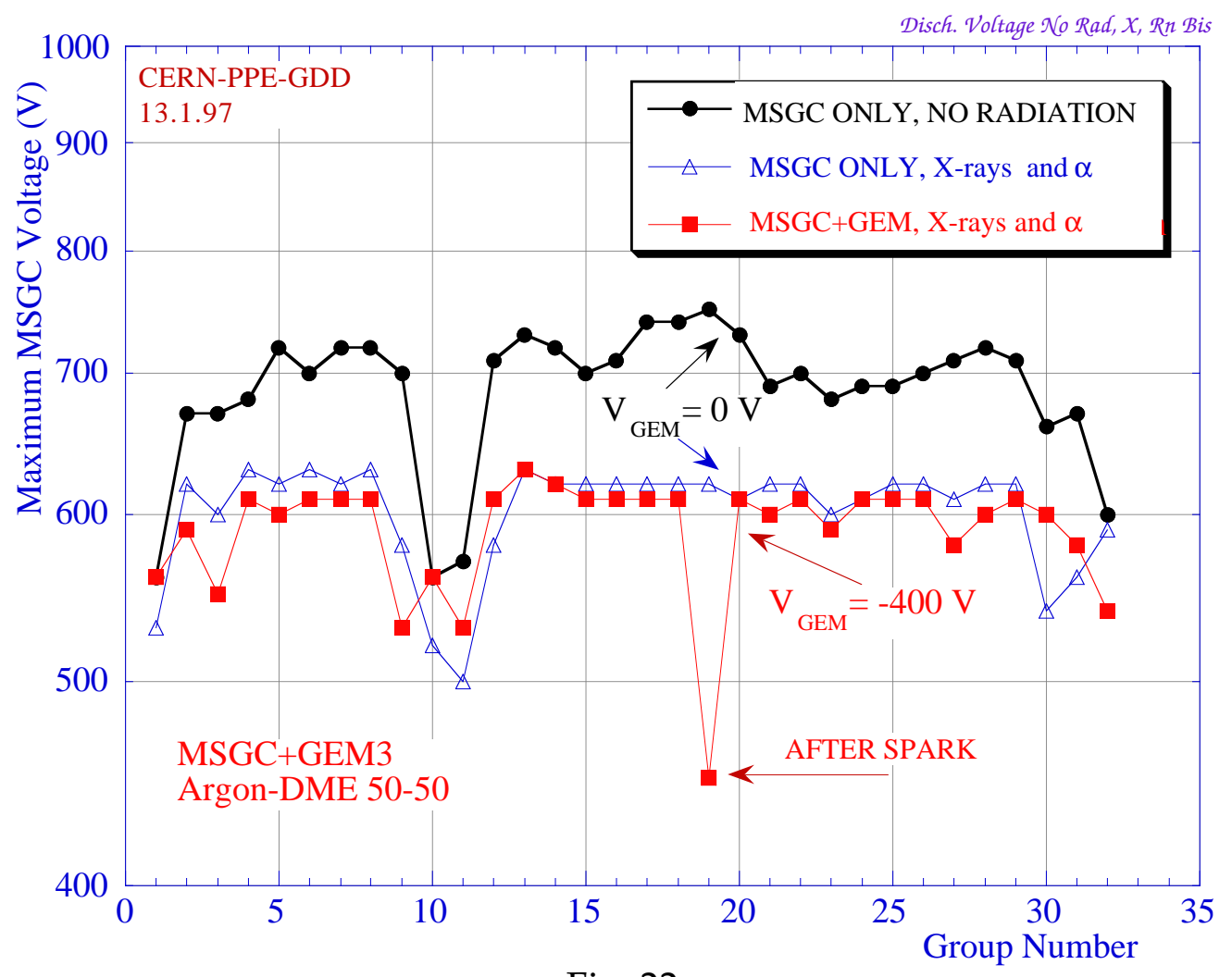

Fig. 22

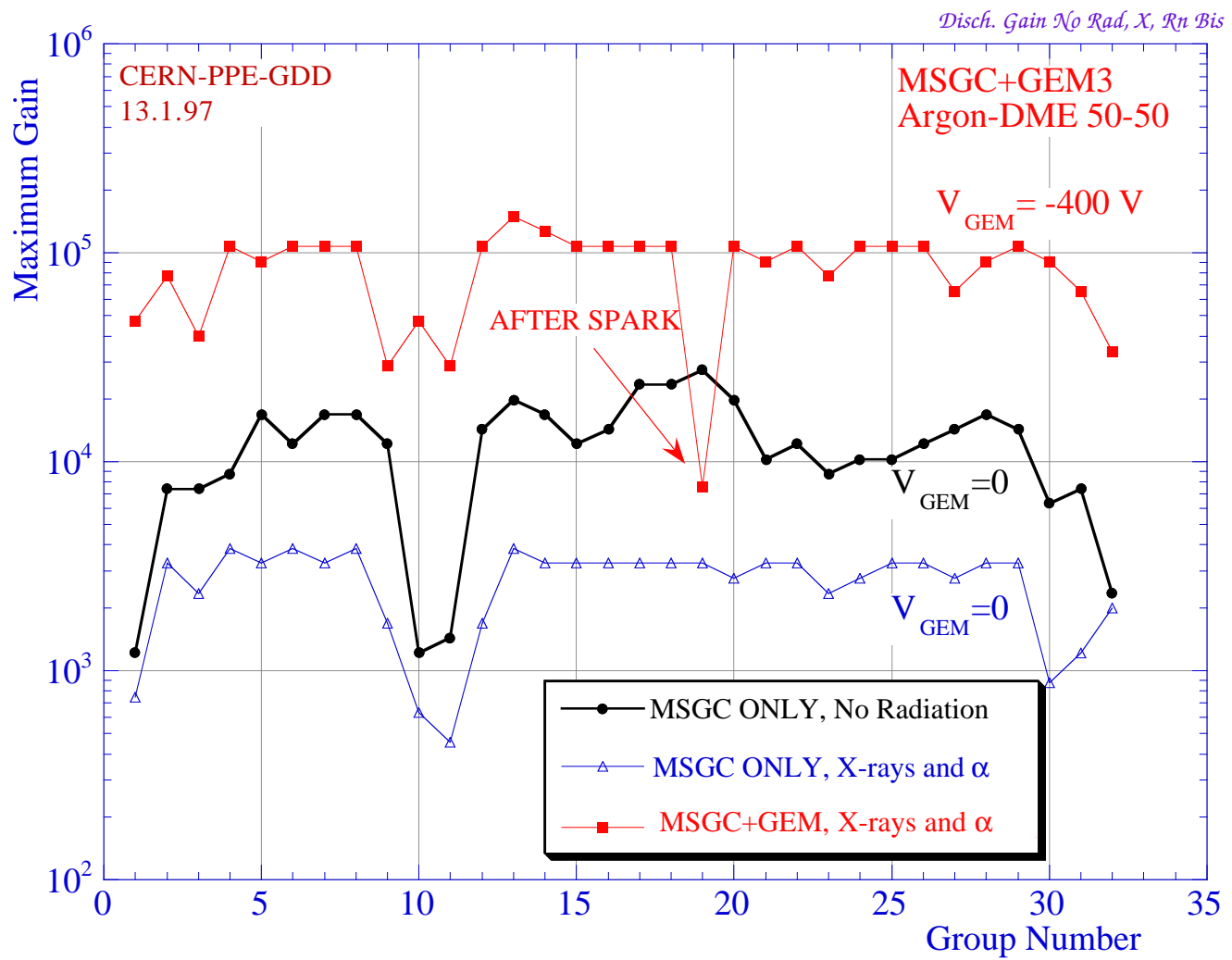

Fig. 23 